\title{
A Jump Diffusion Model for Option Pricing with Three Properties: Leptokurtic Feature, Volatility Smile, and Analytical Tractability
}

\author{
S. G. Kou* \\ Columbia University
}

First draft November, 1999

\begin{abstract}
Brownian motion and normal distribution have been widely used, for example, in the Black-Scholes-Merton option pricing framework, to study the return of assets. However, two puzzles, emerged from many empirical investigations, have got much attention recently, namely (a) the leptokurtic feature that the return distribution of assets may have a higher peak and two (asymmetric) heavier tails than those of the normal distribution, and (b) an empirical abnormity called "volatility smile" in option pricing. To incorporate both the leptokurtic feature and "volatility smile", this paper proposes, for the purpose of studying option pricing, a jump diffusion model, in which the price of the underlying asset is modeled by two parts, a continuous part driven by Brownian motion, and a jump part with the logarithm of the jump sizes having a double exponential distribution. In addition to the above two desirable properties, leptokurtic feature and "volatility smile", the model is simple enough to produce analytical solutions for a variety of option pricing problems, including options, future options, and interest rate derivatives, such as caps and floors, in terms of the $H h$ function. Although there are many models can incorporate some of the three properties (the leptokurtic feature, "volatility smile", and analytical tractability), the current model can incorporate all three under a unified framework.
\end{abstract}

\section{Introduction}

Brownian motion and normal distribution have been widely used to study option pricing and the return of assets; for references, see, for example, Cox and Rubinstein (1985), Duffie (1995), Hull (1999), Ingersoll (1988), Karatzas and Shreve (1998), Merton (1990), Musiela and Rutkowski (1997), Elliot and Kopp (1998), and Boyle, Broadie, and Glasserman (1997). Option pricing papers within the classical Black-Scholes-Merton model that are particularly relevant to the

*312 Mudd Building, Department of IEOR, Columbia University, New York, NY 10027, e-mail: kou@ieor.columbia.edu. The mathematica code used in the current paper can be downloaded from the web page www.ieor.columbia.edu/ ${ }^{\sim}$ kou 
current paper are: Black and Scholes (1973) model for the call and put options; Black (1976) model for options on futures contracts; Heath, Jarrow and Morton (1992) model for options on bonds; and Brace, Gatarek, and Musiela model (1997) for caps and floors, which are options on discretely compounded simple interest rates and are among the most liquated interest rate options (see also Miltersen, Sandmann and Sondermann, 1997, and Jamshidian, 1997).

Despite the successes of Black-Scholes-Merton model based on Brownian motion and normal distribution, two puzzles, emerged from many empirical investigations, have got much attention recently.

(1). The leptokurtic and asymmetric features. In the above classical models, the marginal distribution of the underlying assets is assumed to be normal. However, many empirical studies suggest that the distribution is skewed to the left, and has a higher peak and two heavier tails than those of the normal distribution.

(2). The volatility smile. More precisely, if the Black-Scholes-Merton model is correct, then the implied volatility should be constant; but it is widely recognized that the implied volatility curve resembles a "smile", meaning it is a convex curve of the strike price.

Many researches have been conducted to modify the Black-Scholes models to explain the two puzzles. To incorporate the leptokurtic and asymmetric features, a variety of models have been proposed, including, among others, (a) chaos theory, fractal Brownian motion, and stable processes; see, for example, Mandelbrot (1963, 1967), Mandelbrot, Fisher, and Calvet (1997), Fama (1963, 1965), Rogers (1997), Willinger, Taqqu, and Teverovsky (1999), Samorodnitsky and Taqqu (1994), Peters (1991, 1994); (b) generalized hyperbolic models, including log $t$-model, log hyperbolic model, and log variance gamma model; see, for example, Madan and Seneta (1990), Eberlein and Keller (1995), Barndorff-Nielsen (1995), Praetz (1972), Blattberg and Gonedes (1974); (c) time changed Brownian motions; see, for example, Clark (1973), Andersen (1996), Hurst, Platen and Rachev (1997), Geman, Madan, and Yor (1998), and Heyde (1999). An immediate problem with these models is that it may be difficult to obtain analytical solutions for the purpose of option pricing; more precisely, they might give some analytical formulae for regular call and put options, but certainly not for interest rate derivatives and exotic options, such as perpetual American options, barrier and lookback options.

In a parallel development, different models are also proposed to incorporate the "volatility smile". Popular ones are (a) stochastic volatility and ARCH models; see, for example, Hull and White (1987), Engle (1982, 1995), White (1980), Gouriéroux (1997); (b) constant elasticity model (CEV) model; see, for example, Cox and Ross (1976), Cox, Ingersoll and Ross (1985), 
Davydov and Linetsky (1999), Andersen and Andreasen (1999); (c) normal jump models, first proposed by Merton (1976) and widely used since then; see, for example, Merton (1990), and Duffie (1995); (d) a numerical procedure called "implied binomial trees"; see, for example, Derman and Kani (1994), Dupire (1994), Rubinstein (1994). Aside from the problem that it might not be easy to find analytical solutions for option pricing, especially for exotic options (such as perpetual American options, barrier and lookback options), these models may not produce the leptokurtic and asymmetric features, especially the "high peak" feature.

The current paper attempts to propose a new model, which has three properties.

- It has the leptokurtic and asymmetric features, under which the return distribution of the assets has a higher peak and two heavier tails than the normal distribution, especially the left tail; see section 2 .

- It leads to analytical solutions to many option pricing problems, including

- call and put options, and options on futures; see section 4.

- interest rate derivatives, such as caplets, caps, and bond options; see section 5 .

- exotic options, such as perpetual American options, barrier and lookback options, which will be reported in a separate paper.

- It can reproduce the "volatility smile"; see section 5.2 .

Although there are, as we discussed before, many models that can incorporate some of the three properties (the leptokurtic feature, analytically tractability, and "volatility smile"), the current model can incorporate all three under a unified framework.

The model that we propose for the price of an underlying asset (for example, a stock or a stock index) is very simple. It consists of two parts, a continuous part modeled by a geometric Brownian motion, and a jump part, with the logarithm of the jump sizes having a double exponential distribution and the jump times corresponding to the event times of a Poisson process. Because of the simplicity, the parameters in the model can be easily interpreted, and the closed form solutions for option pricing can be obtained in terms of the $H h$ functions.

General properties of jump diffusion models with independent identically distributed jump sizes have been extensively studied since the original paper of Merton (1976); for excellent surveys, see Duffie (1995) and Merton (1990). In addition to the modeling and studying of the leptokurtic feature and "volatility smile", the technical contribution of the current paper is 
that we provide an explicit calculation of option prices in the case of the logarithm of the jump sizes being double exponentially distributed. The explicit calculation is made possible partly because of the memoryless property of the double exponential distribution.

The paper is organized in the following way. In section 2 , the model is proposed and the leptokurtic feature is studied. Some preliminary results, including the $H h$ functions, are given in section 3. Formulae for option pricing problems, including options on futures, are provided in section 4. Section 5.1 studies the pricing of interest rate options, such as caplets and bond options. "Volatility smiles" is studied in section 5.2. The last section discusses the advantages and disadvantages of the model.

\section{The model}

The model that we propose for the price of an underlying asset (for example a stock or a stock index) consists of two parts, a continuous part modeled by a geometric Brownian motion, and a jump part, with the logarithm of the jump sizes having a double exponential distribution and the jump times corresponding to the event times of a Poisson process. More precisely, the following stochastic differential equation is used to model the asset price, $S(t)$,

$$
\frac{d S(t)}{S(t)}=\mu d t+\sigma d W(t)+d\left(\sum_{i=1}^{N(t)}\left(V_{i}-1\right)\right)
$$

where $W(t)$ is a standard Wiener process, $N(t)$ a Poisson process with rate $\lambda$, and $\left\{V_{i}\right\}$ a sequence of independent identically distributed (i.i.d.) nonnegative random variables such that $X=\log (V)$ has a double exponential distribution with the density

$$
f_{X}(x)=\frac{1}{2 \eta} e^{-|x-\kappa| / \eta}, \quad 0<\eta<1
$$

In other words,

$$
X-\kappa=\left\{\begin{array}{ll}
\xi, & \text { with probability } 1 / 2 \\
-\xi, & \text { with probability } 1 / 2
\end{array}\right\},
$$

where $\xi$ is an exponential random variable with mean $\eta$ and variance $\eta^{2}$. All sources of randomness, $N(t), W(t)$, and $X$ 's, are assumed to be independent.

Remark. For notation simplicity, and in order to get some analytic solutions for various option pricing problems, here the drift $\mu$ and the volatility $\sigma$ are assumed to be constants, and the Wiener processes and jumps are assumed to be one-dimensional. These assumptions, however, can be easily dropped for the purpose of developing a general theory. 
Solving the stochastic different equation (2.1) gives the dynamics of the asset price as follows:

$$
S(t)=S(0) \exp \left\{\left(\mu-\frac{1}{2} \sigma^{2}\right) t+\sigma W(t)\right\} \prod_{i=1}^{N(t)} V_{i} .
$$

Merton (1976) first considered the jump diffusion models similar to (2.1) and (2.3). In that paper $X$ 's are assumed to have normal distribution rather than the double exponential distribution, although some general properties, for the models with arbitrary distributions, were discussed there. A major goal of this paper is to show that, within this very simple jump diffusion framework, it is possible to get some desirable features of the return of the asset, such as higher peak and heavier tails, particularly the left tail, as well as retaining analytic tractability of the model, so that options can, in terms of the $H h$ functions, be priced in closed form.

To motive further studies of the model, it would be of interest to discuss the return of the underlying asset in such a model. Using (2.3), we get

$$
\begin{aligned}
\frac{\Delta S(t)}{S(t)} & =\frac{S(t+\Delta t)}{S(t)}-1 \\
& =\exp \left\{\left(\mu-\frac{1}{2} \sigma^{2}\right) \Delta t+\sigma(W(t+\Delta t)-W(t))+\sum_{i=N(t)+1}^{N(t+\Delta t)} X_{i}\right\}-1
\end{aligned}
$$

where a summation over an empty set is taken to be zero. If the time interval $\Delta t$ is small, as in the case of daily observations, the return can be approximated, ignoring the terms with order higher than $\Delta t$ and using the expansion $e^{x} \approx 1+x+x^{2} / 2$, by

$$
\begin{aligned}
\frac{\Delta S(t)}{S(t)} & \approx\left(\mu-\frac{1}{2} \sigma^{2}\right) \Delta t+\sigma(W(t+\Delta t)-W(t))+\sum_{i=N(t)}^{N(t+\Delta t)} X_{i}+\frac{1}{2} \sigma^{2}(W(t+\Delta t)-W(t))^{2} \\
& \approx \mu \Delta t+\sigma Z \sqrt{\Delta t}+\sum_{i=N(t)+1}^{N(t+\Delta t)} X_{i},
\end{aligned}
$$

where $Z$ is a standard normal random variable. Notice that the probability of the Poisson process $N(t)$ having one jump is $\lambda \Delta t$, and the probability of having more than one jumps is $o(\Delta t)$. Therefore, if $\lambda \Delta t$ is small, ignoring multiple jumps leads to

$$
\sum_{i=N(t)+1}^{N(t+\Delta t)} X_{i} \approx\left\{\begin{array}{ll}
X_{i} & \text { with prob. } \lambda \Delta t \\
0 & \text { with prob. } 1-\lambda \Delta t
\end{array}\right\} .
$$

In summary, for small $\Delta t$, the return can be approximated, in distribution, by

$$
\frac{\Delta S(t)}{S(t)} \approx \mu \Delta t+\sigma Z \sqrt{\Delta t}+B \cdot X
$$


where $B$ is a Bernoulli random variable with $P(B=1)=\lambda \Delta t$ and $P(B=0)=1-\lambda \Delta t$, and $X$ is given by (2.2). Notice that dropping the last term in (2.4) leads to the classical model of geometric Brownian motion, with the return, $\Delta S(t) / S(t)$, being characterized approximately by a normal density.

By using the result (3.16), in section 3, of the density of sum of double exponential and the normal random variable, we have that the density, $g$, of the right hand side of (2.4), being an approximation for the return $\frac{\Delta S(t)}{S(t)}$, is given by

$$
\begin{gathered}
g(x)=\frac{\lambda \Delta t}{2 \eta} e^{\sigma^{2} \Delta t /\left(2 \eta^{2}\right)}\left\{e^{-(x-\mu \Delta t-\kappa) / \eta} \Phi\left(\frac{(x-\mu \Delta t-\kappa) \eta-\sigma^{2} \Delta t}{\sigma \eta \sqrt{\Delta t}}\right)\right. \\
\left.+e^{(x-\mu \Delta t-\kappa) / \eta} \Phi\left(-\frac{(x-\mu \Delta t-\kappa) \eta+\sigma^{2} \Delta t}{\sigma \eta \sqrt{\Delta t}}\right)\right\} \\
+(1-\lambda \Delta t) \frac{1}{\sigma \sqrt{\Delta t}} \varphi\left(\frac{x-\mu \Delta t}{\sigma \sqrt{\Delta t}}\right),
\end{gathered}
$$

where $\varphi(\cdot)$ is the standard normal density function and $\kappa=E(X)$. The density $g$ has the mean and the variance given by

$$
\begin{gathered}
E_{g}(G)=\mu \Delta t+\lambda \Delta t \kappa \\
\operatorname{Var}_{g}(G)=\sigma^{2} \Delta t+2 \eta^{2} \lambda \Delta t+\kappa^{2} \lambda \Delta t(1-\lambda \Delta t) .
\end{gathered}
$$

Remark. An important feature of this density is that, comparing to the normal density with the identical mean and variance, it has a higher peak around the mean, and two heavier tails, or, in short, the leptokurtic feature. The other thing, worthy of mentioning, is that the density is not symmetric, if the mean jump size $\kappa$ is not zero; in fact, it is skewed to the left if $\kappa<0$, and skewed to the right if $\kappa>0$. These features have been favored by many empirical investigations.

Below are figures of the density, $g(x)$, compared with the normal density with the same mean and variance given by (2.5) and (2.6). The first figure compares the overall shapes of the two densities, the second one details the shapes around the peak areas, and the last two show the left and right tails. The dot line is used for the normal density, and the solid line is used for the model. The parameters used here are $\Delta t=1$ day $=1 / 250$ year, $\sigma=20 \%$ per year, $\mu=15 \%$ per year, $\lambda=10$ per year, $\kappa=-2 \%, \eta=2 \%$. In other words, there are about 10 jumps per year with average jump size $-2 \%$, and the jump volatility $2 \%$. The jump parameters used here seem to be quite reasonable, if not conservative, for a U. S. stock. The leptokurtic feature, however, is quite evident. The peak of the density $g$ is about 30.6, whereas that of the 
normal density is about 27.7. The density $g$ has heavier tails than the normal density, especially for the left tail, which could reach $-10 \%$ while the normal density is basically confined within $-4 \%$.

Remark. Additional numerical plots suggest that the feature of higher peak and heavier tails becomes more significant if either $|\kappa|$ (the jump size) or $\eta$ (the jump volatility) increases.

Remark. Although it is possible to get heavier tails by using the normal distribution for the logarithm of the jump sizes, instead of the double exponential distribution, it is impossible for it to have both high peak and heavier tails.

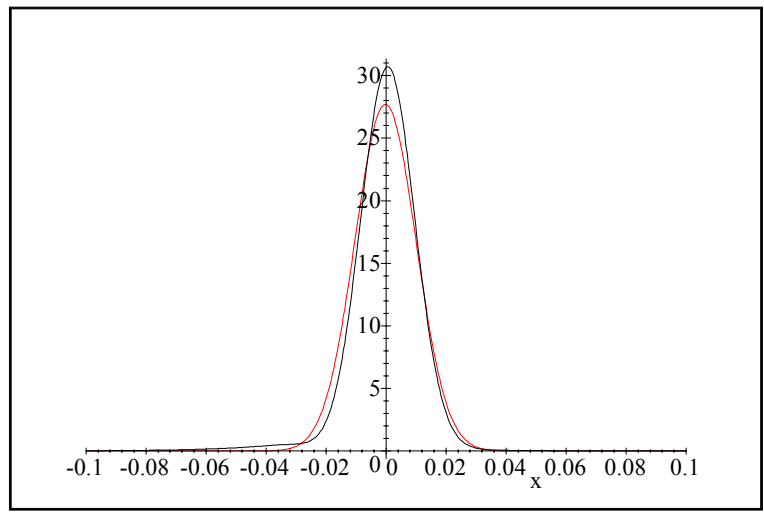

Figure 2.1: Overall comparison

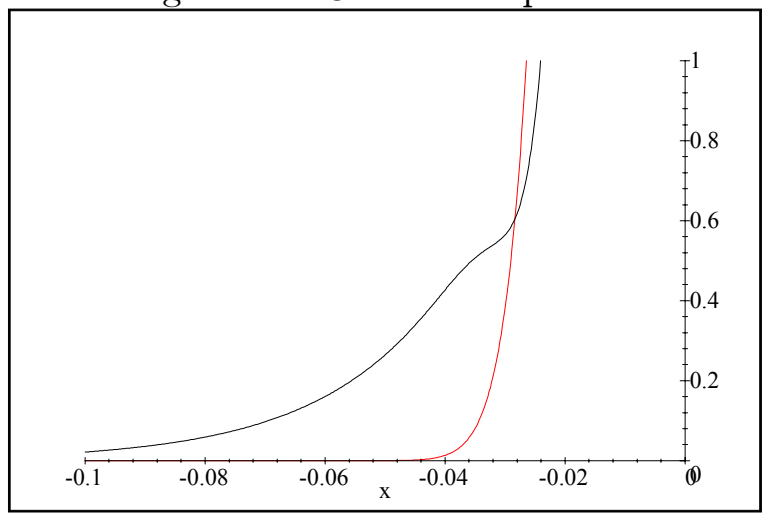

Figure 2.3: Left tail comparison

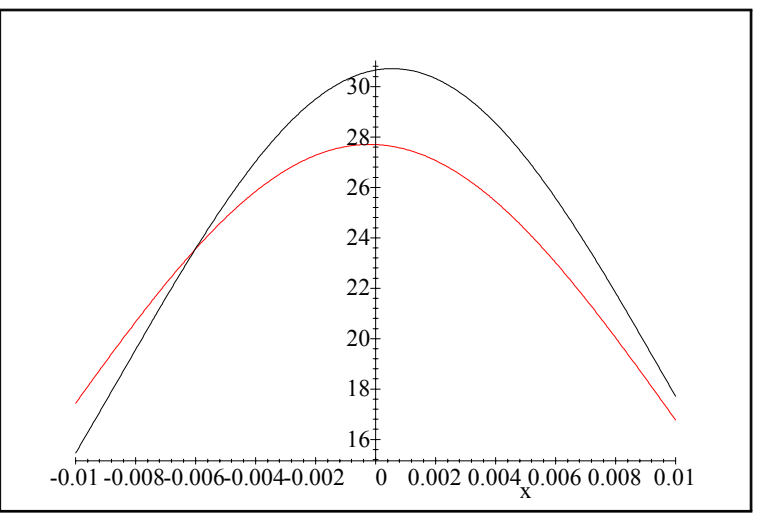

Figure 2.2: Peak comparison

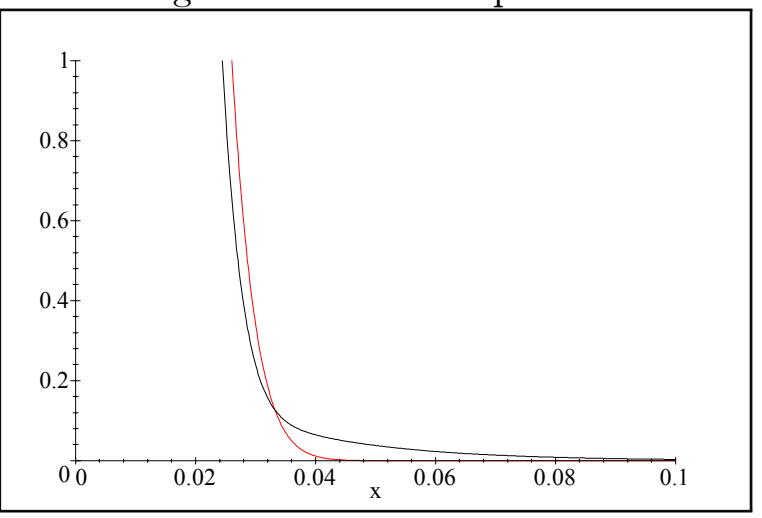

Figure 2.4: Right tail comparison

\section{Some preliminary results}

To price options, we have to notice that our jump diffusion model leads to an incomplete market; therefore, the standard hedging arguments may not be useful here. However, as we mentioned before, many papers have studied the general properties of various jump diffusion models. In 
particular, standard results tell us that (see, for example, Duffie 1995, and Merton, 1990), for a given set of risk premiums, we can consider a risk-neutral measure $\mathrm{P}^{*}$

$$
\begin{aligned}
\frac{d S(t)}{S(t)} & =(r-\lambda E(V-1)) d t+\sigma d W(t)+d\left(\sum_{i=1}^{N(t)}\left(V_{i}-1\right)\right) \\
& =(r-\lambda \zeta) d t+\sigma d W(t)+d\left(\sum_{i=1}^{N(t)}\left(V_{i}-1\right)\right),
\end{aligned}
$$

where $\zeta=\frac{e^{\kappa}}{1-\eta^{2}}-1,0<\eta<1$, via (3.3); and the parameters, $\kappa, \eta, \lambda$, and $\sigma$, here are no longer physical parameters, but the risk-neutral parameters taking consideration also of the risk premiums. The unique strong solution of the above equation is given by

$$
S(t)=S(0) \exp \left\{\left(r-\frac{1}{2} \sigma^{2}-\lambda \zeta\right) t+\sigma W(t)\right\} \prod_{i=1}^{N(t)} V_{i}
$$

For pricing of European options in the jump diffusion model, we need to compute the expectation, under the measure $\mathrm{P}^{*}$, of the discounted final payoff of the option. In particular, the price of a call option at time $0, \psi_{c}(0)$, is given by

$$
\begin{aligned}
\psi_{c}(0) & =\mathrm{E}^{*}\left(e^{-r T} \psi_{c}(T)\right) \\
& =\mathrm{E}^{*}\left(e^{-r T}\left(S(0) \exp \left\{\left(r-\frac{\sigma^{2}}{2}-\lambda \zeta\right) T+\sigma \sqrt{T} Z\right\} \prod_{j=1}^{N(T)} V_{j}-K\right)^{+}\right)
\end{aligned}
$$

where $\psi_{c}(T)=(S(T)-K)^{+}$and $Z$ is a standard normal random variable. Notice the fact, which will be used later, that under this measure $\mathrm{P}^{*}$

$$
\begin{aligned}
\mathrm{E}^{*}\left(e^{-r T} S(T)\right) & =e^{-r T} S(0) \mathrm{E}^{*}\left\{\exp \left\{\left(r-\frac{1}{2} \sigma^{2}-\lambda \zeta\right) t+\sigma W(t)\right\} \prod_{i=1}^{N(t)} V_{i}\right\} \\
& =S(0) \mathrm{E}^{*}\left\{\exp \{-\lambda \zeta t\} \prod_{i=1}^{N(t)} V_{i}\right\} \\
& =S(0) \exp \{-\lambda \zeta t\} \sum_{n=0}^{\infty} \mathrm{E}^{*}\left\{\prod_{i=1}^{n} V_{i}\right\} \frac{(\lambda t)^{n}}{n !} e^{-\lambda t} \\
& =S(0) \exp \{-\lambda \zeta t\} \sum_{n=0}^{\infty}(\zeta+1)^{n} \frac{(\lambda t)^{n}}{n !} e^{-\lambda t} \\
& =S(0) \exp \{-\lambda \zeta t\} e^{\lambda(\zeta+1) t} e^{-\lambda t},
\end{aligned}
$$

in other words,

$$
\mathrm{E}^{*}\left(e^{-r T} S(T)\right)=S(0)
$$

justifying the name "risk-neutral". 


\subsection{Double exponential distribution}

The double exponential density, defined by

$$
f_{X}(x)=\frac{1}{2 \eta} e^{-|x-\kappa| / \eta}, \quad 0<\eta<1
$$

was proposed by Laplace (1774), giving rise to another name - " the first law of Laplace", while the "second law of Laplace" is the normal density. It can also be represented as

$$
X-\kappa=\left\{\begin{array}{ll}
\xi & \text { with probability } 1 / 2 \\
-\xi & \text { with probability } 1 / 2
\end{array}\right\}
$$

where $\xi$ is an exponential random variable with mean $\eta$ and variance $\eta^{2}$. One of the most important properties of this density is that it has the leptokurtic feature, namely it has a higher peak and two heavier tails than those of the normal density with the identical mean and variance; see, for example, Kotz, Johnson, and Balakrishnan (1995).

Remark. A unique feature, inherited from the exponential distribution, about the double exponential distribution is the memoryless property, to be used in the next subsection. It is because of this memoryless property, closed form solutions for various option pricing problems are feasible.

Although, at the point of the mean, the double exponential density has a discontinuity of the derivative, in our model of the option pricing it will be smoothed out by the normal distribution from the continuous Brownian motion part, resulting in a density everywhere differentiable.

A useful result of the double exponential random variable is that

$$
E\left(e^{X}\right)=\frac{e^{\kappa}}{1-\eta^{2}} \equiv \zeta+1,0<\eta<1
$$

since

$$
\int_{-\infty}^{0} e^{x} \frac{1}{2 \eta} e^{x / \eta} d x+\int_{0}^{\infty} e^{x} \frac{1}{2 \eta} e^{-x / \eta} d x=\frac{1}{2 \eta}\left(\frac{1}{1+\frac{1}{\eta}}+\frac{1}{\frac{1}{\eta}-1}\right)=\frac{1}{1-\eta^{2}} .
$$

Remark. In a forthcoming paper by Heyde and Kou (2000) they point out that, for a sample size of 5000 (20-years-daily data), it is very difficult to distinguish the double exponential distribution from the power type distributions, such as $t$-distribution; and, in fact, they also show that many statistical procedures may fail to detect differences between the double exponential distribution and $t$-distributions. For example, the following histograms suggest that they are almost indifferent to human eyes; for details, see Heyde and Kou (2000). It should be emphasized, however, that in the current paper we are not arguing which one is more suitable for fitting empirical observations, but just to say that, since the $t$-distribution 
cannot, in general, give closed form solutions, the double exponential distribution may be a good alternative choice as a balance between reality and analytical tractability; furthermore, $t$-distribution cannot have the high peak feature.

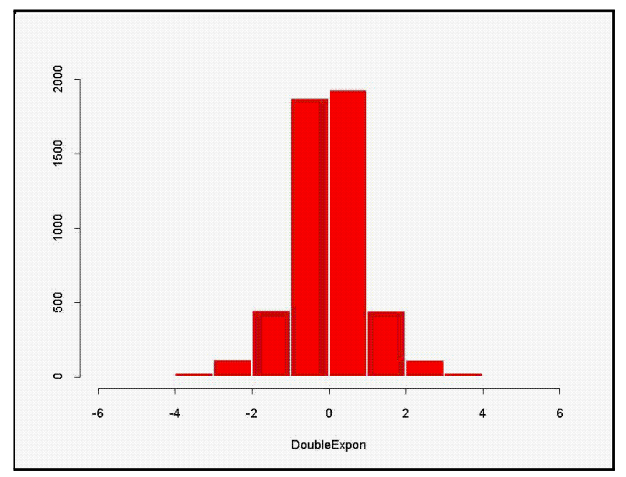

Figure 3.1: Histogram of the double exponential distribution with mean 0 and variance 1.

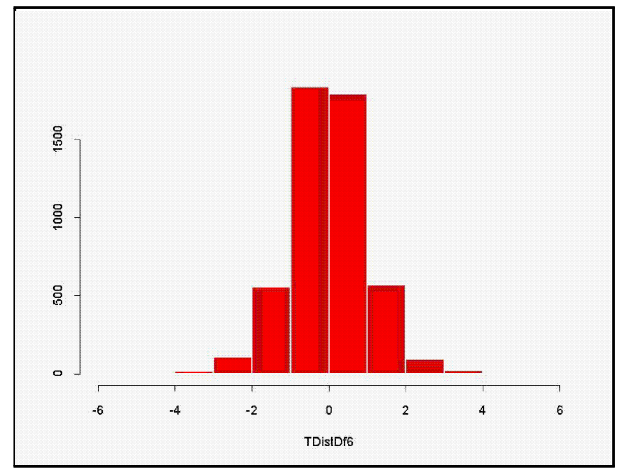

Figure 3.2: Histogram of the t-distribition with d.f. 6 .

\subsection{Sum of double exponential random variables}

Let $X_{i}, i=1,2, \ldots$, be a sequence of independent identically distributed (i.i.d.) double exponential random variables with

$$
X-\kappa \stackrel{d}{=}\left\{\begin{array}{ll}
\xi, & \text { with probability } 1 / 2 \\
-\xi, & \text { with probability } 1 / 2
\end{array}\right\},
$$

where $\xi$ is an exponential random variable with mean $\eta$ and variance $\eta^{2}$. Notice that

$$
E(X)=\kappa, \quad \operatorname{Var}(X)=2 \eta^{2} .
$$


Remark. The notation $\stackrel{d}{=}$ means equal in distribution. Without further notice, $\xi_{i}, \tilde{\xi}_{i}, \xi$, and $\tilde{\xi}$ denote i.i.d. exponential random variables with mean $\eta$ and variance $\eta^{2}$.

By the memoryless property, we have the conditional distribution

$$
\left(\xi_{1}-\xi_{2} \mid \xi_{1}>\xi_{2}\right) \stackrel{d}{=} \xi, \quad\left(\xi_{1}-\xi_{2} \mid \xi_{1}<\xi_{2}\right) \stackrel{d}{=}-\xi
$$

thus leading to the conclusion that

$$
\xi_{1}-\xi_{2} \stackrel{d}{=}\left\{\begin{array}{ll}
\xi, & \text { with probability } 1 / 2 \\
-\xi, & \text { with probability } 1 / 2
\end{array}\right\},
$$

because the probabilities of the events $\xi_{1}>\xi_{2}$ and $\xi_{1}<\xi_{2}$ are equal. Therefore, we have

$$
X_{1}+X_{2}-2 \kappa \stackrel{d}{=}\left\{\begin{array}{ll}
\xi_{1}+\xi_{2}, & 1 / 4 \\
\xi_{1}-\xi_{2}, & 1 / 4 \\
-\xi_{1}+\xi_{2}, & 1 / 4 \\
-\xi_{1}-\xi_{2}, & 1 / 4
\end{array}\right\} \stackrel{d}{=}\left\{\begin{array}{ll}
\xi_{1}+\xi_{2}, & 1 / 4 \\
\xi_{1}, & 1 / 4 \\
-\xi_{1}, & 1 / 4 \\
-\xi_{1}-\xi_{2}, & 1 / 4
\end{array}\right\} ;
$$

in other words,

$$
X_{1}+X_{2}-2 \kappa \stackrel{d}{=}\left\{\begin{array}{ll}
\sum_{i=1}^{M} \xi_{i}, & \text { with probability } 1 / 2 \\
-\sum_{i=1}^{M} \xi_{i}, & \text { with probability } 1 / 2
\end{array}\right\},
$$

where $\xi_{i}$ are i.i.d. exponential random variables, and $M$ is a discrete random variable with $P(M=1)=1 / 2$, and $P(M=2)=1 / 2$. Similar computation leads to

$$
X_{1}+X_{2}+X_{3}-3 \kappa \stackrel{d}{=}\left\{\begin{array}{ll}
\sum_{i=1}^{M} \xi_{i}, & \text { with probability } 1 / 2 \\
-\sum_{i=1}^{M} \xi_{i}, & \text { with probability } 1 / 2
\end{array}\right\},
$$

where $M$ is a discrete random variable with $P(M=3)=1 / 4, P(M=2)=3 / 8$, and $P(M=$ $1)=3 / 8$. The following proposition extends (3.5) and (3.6).

Proposition 1. We have that, for every $n \geq 1$, the following decomposition

$$
\sum_{i=1}^{n} X_{i}-n \kappa \stackrel{d}{=}\left\{\begin{array}{cc}
\sum_{i=1}^{M(n)} \xi_{i}, & \text { with probability } 1 / 2 \\
-\sum_{i=1}^{M(n)} \xi_{i}, & \text { with probability } 1 / 2
\end{array}\right\},
$$

where $\xi_{i}$ are i.i.d. exponential random variables with mean $\eta$, and $M(n)$ is a discrete random variable, independent of the $X$ 's, with

$$
P(M(n)=j)=\frac{2^{j}}{2^{2 n-1}}\left(\begin{array}{c}
2 n-j-1 \\
n-1
\end{array}\right), 1 \leq j \leq n,
$$

where $\left(\begin{array}{l}0 \\ 0\end{array}\right)$ is defined to be 1 .

Proof. See the appendix. 
As a key step in deriving closed form solutions, this proposition indicates that the sum of i.i.d. double exponential random variables can be written, in distribution, as a single (randomly) mixed double gamma random variable. This decomposition simplifies the calculation enormously, because, by conditioning on $M(n)$, we have only one gamma random variable to deal with. A general result similar to the decomposition (3.7) was first discovered in Shanthikumar (1985), although (3.8) gives a more explicit result for the distribution of $M(n)$. Furthermore, the proofs are totally different: a combinatorial approach is used here, while the proof in Shanthikumar (1985) is based on the Laplace transform.

\subsection{Hh functions}

For option pricing, it is necessary to consider the distribution of the sum of normal and double exponential (or gamma) random variables. Fortunately, this distribution can be obtained in closed form, thanks to a special function, $H h$ function, from the mathematical physics literature. The definition of the $H h$ function, for example on p. 691 of Abramowitz and Stegun (1972), is given by

$$
\begin{gathered}
\operatorname{Hh}_{n}(x)=\int_{x}^{\infty} \operatorname{Hh}_{n-1}(y) d y=\frac{1}{n !} \int_{x}^{\infty}(t-x)^{n} e^{-t^{2} / 2} d t \geq 0, \quad n=0,1,2, \ldots \\
\frac{d}{d x} \operatorname{Hh}_{n}(x)=-\operatorname{Hh}_{n-1}(x), \quad n=0,1,2, \ldots
\end{gathered}
$$

and

$$
\operatorname{Hh}_{-1}(x)=e^{-x^{2} / 2}=\sqrt{2 \pi} \varphi(x), \operatorname{Hh}_{0}(x)=\sqrt{2 \pi} \Phi(-x) .
$$

Therefore, the $H h$ function can be viewed as a generalization of the cumulative normal distribution function. Notice that, for a standard normal random variable $Z$,

$$
E\left((Z-x)^{n} I(Z \geq x)\right)=\frac{n !}{\sqrt{2 \pi}} \operatorname{Hh}_{n}(x), \quad n=0,1,2, \ldots
$$

Furthermore, for every $n \geq 0$, the $H h$ function is a non-increasing function such that

$$
\begin{aligned}
& \operatorname{Hh}_{n}(x) \rightarrow 0, \text { as } x \rightarrow \infty, \quad n=-1,0,1,2, \ldots, \\
& \operatorname{Hh}_{n}(x) \rightarrow \infty, \text { as } x \rightarrow-\infty, \quad n=1,2,3, \ldots \\
& \operatorname{Hh}_{0}(x)=\sqrt{2 \pi} \Phi(-x) \rightarrow \sqrt{2 \pi}, \text { as } x \rightarrow-\infty
\end{aligned}
$$

There is also an interesting connection between the $H h$ function and the parabolic cylinder function, $U$, which is widely used in mathematical physics:

$$
U\left(n+\frac{1}{2}, x\right)=e^{x^{2} / 4} \operatorname{Hh}_{n}(x) .
$$


Notice that if $x<0$ then the integrand in (3.9) has a maxima at

$$
\frac{x+\sqrt{x^{2}+4 n}}{2}
$$

and most of the value of the integral is realized around the peak area. Therefore, for numerical computation, we want to split the integral more around the peak area.

The $H h$ function can be computed, fast and easily, by evaluating the integral in (3.9) directly using software packages, such as Mathematica, Maple, Splus, or Matlab. Alternatively, it can also be obtained by using a table in Abramowitz and Stegun (1972) for the parabolic cylinder functions $U$, along with (3.11). The following is a mathematica code for the $H h$ function. Notice in the code the function is set to be zero if $x \geq 10$, because the value is less than $10^{-100}$. In addition, we only need more interval spliting if $x<-6$.

$\operatorname{Hh}\left[\mathrm{x}_{-}, \mathrm{n}_{-}\right]:=\operatorname{If}[\mathrm{x}>=-6$, If $[\mathrm{x}<10$, $1 / \mathrm{n} ! * \mathrm{NIntegrate}[(\mathrm{t}-\mathrm{x}) \wedge \mathrm{n} * \operatorname{Exp}[-\mathrm{t} \wedge 2 / 2],\{t, \mathrm{x}$, Infinity $\}], 0]$, $($ temp $=(\mathrm{x}+\operatorname{Sqrt}[\mathrm{x} * \mathrm{x}+4 * \mathrm{n}]) * 0.5$;

( NIntegrate $[(\mathrm{t}-\mathrm{x}) \wedge \mathrm{n} * \operatorname{Exp}[-\mathrm{t} \wedge 2 / 2],\{\mathrm{t}, \mathrm{x}, \mathrm{temp}-3\}]+$

NIntegrate $[(\mathrm{t}-\mathrm{x}) \wedge \mathrm{n} * \operatorname{Exp}[-\mathrm{t} \wedge 2 / 2],\{\mathrm{t}$, temp-3, temp-1 $\}]+$

NIntegrate $[(t-x) \wedge n * \operatorname{Exp}[-t \wedge 2 / 2],\{t, t e m p-1$, temp $\}]+$

NIntegrate $[(t-x) \wedge n * \operatorname{Exp}[-t \wedge 2 / 2],\{t$, temp, temp +1$\}]+$

NIntegrate $[(t-x) \wedge n * \operatorname{Exp}[-t \wedge 2 / 2],\{t$, temp +1, temp +3$\}]+$

NIntegrate $[(t-x) \wedge n * \operatorname{Exp}[-\mathrm{t} \wedge 2 / 2],\{\mathrm{t}, \mathrm{temp}+3$, Infinity $\}]) / \mathrm{n} !)]$

The following are some pictures for the $H h$ function. It takes only about 15 seconds to generate all the pictures on a Pentium 400 by using the software package Maple.

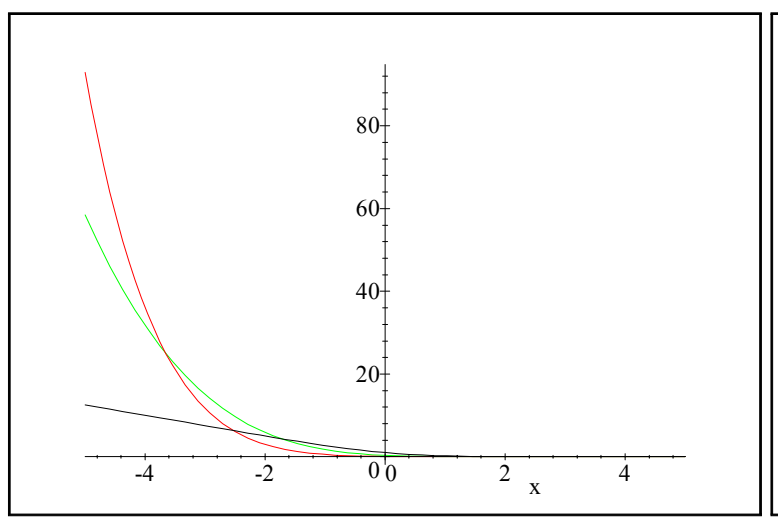

Figure 3.3: $H h$ functions for $n=1,3,5$.

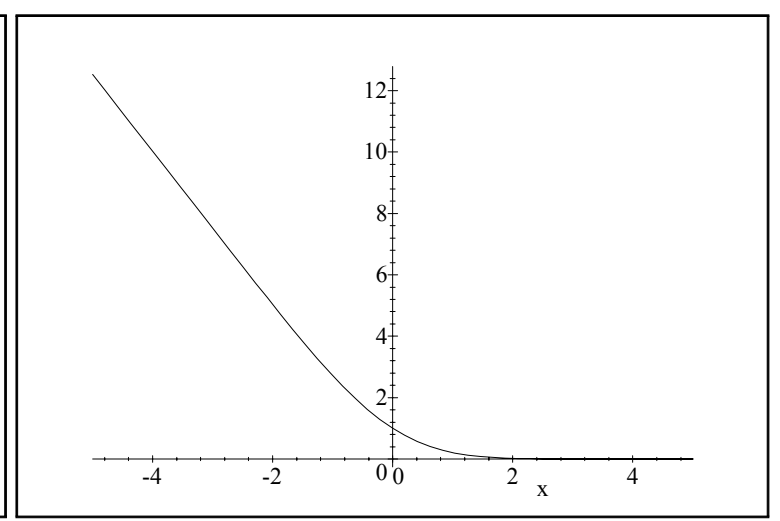

Figure 3.4: $n=1$. 


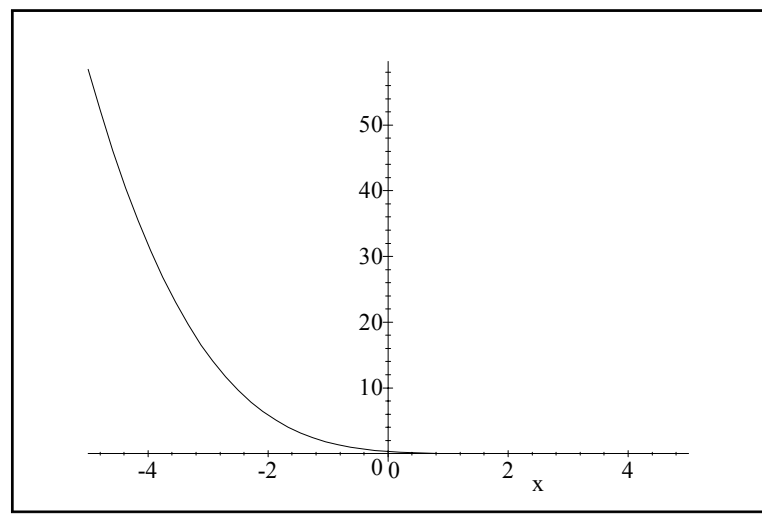

Figure 3.5: $n=3$.

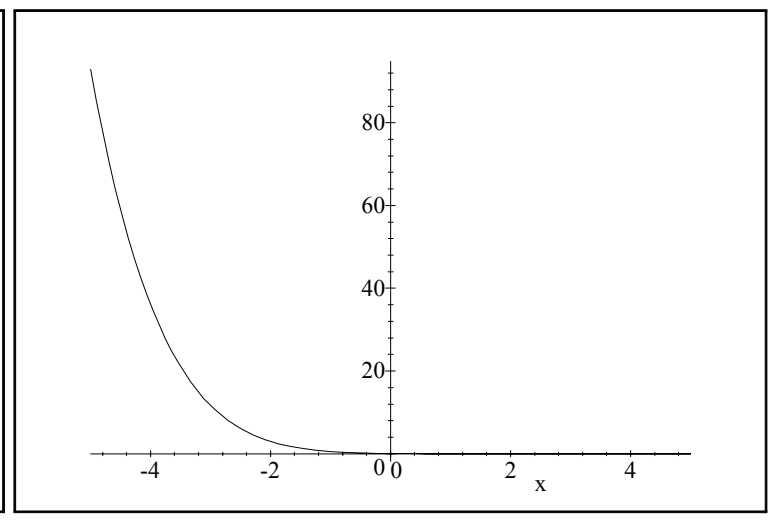

Figure 3.6: $n=5$.

The $H h$ function can be computed very fast. As a hypothetical example, though not relevant to the finance problems we are studying, it only takes a fractional CPU time on a Pentium 400 to get that $H h[-1000,100]=2.6992 * 10^{142}$. The following proposition, which will be used later, confirms what are observed from the above pictures about the limiting behavior of the $H h$ function. More precisely, the left tail of an $H h$ function has a polynomial growth rate, and the right tail has an exponential decay rate.

Proposition 2. For every $n \geq-1$, as $x \rightarrow \infty$,

$$
\operatorname{Hh}_{n}(x) \sim \frac{1}{x^{n+1}} e^{-x^{2} / 2} .
$$

and as $x \rightarrow-\infty$,

$$
\operatorname{Hh}_{n}(x)=O\left(|x|^{n}\right)
$$

Proof. See the appendix.

For option pricing it is important to evaluate the integral $I_{n}$,

$$
I_{n}=\int_{\lambda}^{\infty} e^{\alpha x} \operatorname{Hh}_{n}(\beta x-\delta) d x, n \geq 0
$$

for arbitrary constants $\alpha, \lambda$, and $\beta$.

Proposition 3. If $\beta>0$, then, for all $n \geq 0$,

$$
\begin{aligned}
I_{n} & =\int_{\lambda}^{\infty} e^{\alpha x} \operatorname{Hh}_{n}(\beta x-\delta) d x \\
& =-\frac{e^{\alpha \lambda}}{\alpha} \sum_{i=0}^{n}\left(\frac{\beta}{\alpha}\right)^{n-i} \operatorname{Hh}_{i}(\beta \lambda-\delta)+\left(\frac{\beta}{\alpha}\right)^{n+1} \frac{\sqrt{2 \pi}}{\beta} e^{\frac{\alpha \delta}{\beta}+\frac{\alpha^{2}}{2 \beta^{2}}} \Phi\left(-\beta \lambda+\delta+\frac{\alpha}{\beta}\right) .
\end{aligned}
$$

If $\beta<0$ and $\alpha<0$, then, for all $n \geq 0$,

$$
I_{n}=\int_{\lambda}^{\infty} e^{\alpha x} \operatorname{Hh}_{n}(\beta x-\delta) d x
$$




$$
=-\frac{e^{\alpha \lambda}}{\alpha} \sum_{i=0}^{n}\left(\frac{\beta}{\alpha}\right)^{n-i} \operatorname{Hh}_{i}(\beta \lambda-\delta)-\left(\frac{\beta}{\alpha}\right)^{n+1} \frac{\sqrt{2 \pi}}{\beta} e^{\frac{\alpha \delta}{\beta}+\frac{\alpha^{2}}{2 \beta^{2}}} \Phi\left(\beta \lambda-\delta-\frac{\alpha}{\beta}\right) .
$$

If $\beta<0$ and $\alpha>0$, then, for all $n \geq 0$,

$$
I_{n}=\int_{\lambda}^{\infty} e^{\alpha x} \operatorname{Hh}_{n}(\beta x-\delta) d x=\infty .
$$

Proof. See the appendix.

\subsection{Sum of double exponential and the normal random variables}

Let $X$ be a double exponential random variable with density function

$$
f_{X}(x)=\frac{1}{2 \eta} e^{-|x| / \eta} .
$$

Let $Y$ be a standard normal distribution $N\left(0, \sigma^{2}\right)$. A lengthy, though straightforward, calculation shows that the density of $X+Y$ is given by

$$
f_{X+Y}(t)=\frac{1}{\eta} e^{\sigma^{2} /\left(2 \eta^{2}\right)}\left\{\frac{1}{2} e^{-t / \eta} \Phi\left(\frac{t \eta-\sigma^{2}}{\sigma \eta}\right)+\frac{1}{2} e^{t / \eta} \Phi\left(-\frac{t \eta+\sigma^{2}}{\sigma \eta}\right)\right\},
$$

and

$$
P(X+Y \geq u)=\Phi\left(-\frac{u}{\sigma}\right)+\frac{1}{2} e^{-u / \eta} e^{\sigma^{2} /\left(2 \eta^{2}\right)} \Phi\left(\frac{u \eta-\sigma^{2}}{\sigma \eta}\right)-\frac{1}{2} e^{u / \eta} e^{\sigma^{2} /\left(2 \eta^{2}\right)} \Phi\left(\frac{-u \eta-\sigma^{2}}{\sigma \eta}\right) .
$$

Furthermore, for $c<1 / \eta$,

$$
\begin{gathered}
E\left(a e^{b+c(X+Y)}-K\right)^{+} \\
=a e^{b} \exp \left\{\frac{1}{2} \sigma^{2} c^{2}\right\} \Phi(-h / \sigma+\sigma c)\left\{\frac{1 / 2}{1+c \eta}+\frac{1 / 2}{1-c \eta}\right\}-K \Phi\left(-\frac{h}{\sigma}\right) \\
+\frac{1}{2} e^{-h / \eta} e^{\sigma^{2} /\left(2 \eta^{2}\right)} K\left\{\frac{1}{1-c \eta}-1\right\} \Phi\left(\frac{h \eta-\sigma^{2}}{\sigma \eta}\right) \\
+\frac{1}{2} e^{h / \eta} e^{\sigma^{2} /\left(2 \eta^{2}\right)} K\left\{1-\frac{1}{1+c \eta}\right\} \Phi\left(-\frac{h \eta+\sigma^{2}}{\sigma \eta}\right) .
\end{gathered}
$$

More generally, we get the following proposition.

Proposition 4. Let $X$ be a random variable such that

$$
X=\left\{\begin{array}{ll}
\sum_{i=1}^{n} \xi_{i} & \text { with probability } p \\
-\sum_{i=1}^{n} \xi_{i} & \text { with probability } 1-p
\end{array}\right\}
$$

with the density function

$$
f_{X}(x)=p \frac{(1 / \eta) e^{-x / \eta}(x / \eta)^{n-1}}{(n-1) !} I(x \geq 0)+(1-p) \frac{(1 / \eta) e^{x / \eta}(-x / \eta)^{n-1}}{(n-1) !} I(x<0),
$$


and $Y$ be a normal random variable with distribution $N\left(0, \sigma^{2}\right)$. Then, in terms of the Hh function, the density of $X+Y$ is given, for $n \geq 1$, by

$$
f_{X+Y}(t)=\frac{\sigma^{n}}{\eta^{n}} \frac{e^{\sigma^{2} /\left(2 \eta^{2}\right)}}{\sigma \sqrt{2 \pi}}\left\{p e^{-t / \eta} \operatorname{Hh}_{n-1}\left(-\frac{t \eta-\sigma^{2}}{\sigma \eta}\right)+(1-p) e^{t / \eta} \mathrm{Hh}_{n-1}\left(\frac{t \eta+\sigma^{2}}{\sigma \eta}\right)\right\}
$$

for $n \geq 1$,

$$
\begin{aligned}
P(X+Y \geq u) & =\Phi\left(-\frac{u}{\sigma}\right)+p e^{-u / \eta} e^{\sigma^{2} /\left(2 \eta^{2}\right)} \sum_{i=0}^{n-1}\left(\frac{\sigma}{\eta}\right)^{i} \frac{1}{\sqrt{2 \pi}} \operatorname{Hh}_{i}\left(\frac{-u \eta+\sigma^{2}}{\sigma \eta}\right) \\
& -(1-p) e^{u / \eta} e^{\sigma^{2} /\left(2 \eta^{2}\right)} \sum_{i=0}^{n-1}\left(\frac{\sigma}{\eta}\right)^{i} \frac{1}{\sqrt{2 \pi}} \operatorname{Hh}_{i}\left(\frac{u \eta+\sigma^{2}}{\sigma \eta}\right)
\end{aligned}
$$

for $n \geq 1$ and $c<1 / \eta$,

$$
\begin{aligned}
& E\left(a e^{b+c(X+Y)}-K\right)^{+} \\
& =a e^{b}\left(\frac{p}{(1-c \eta)^{n}}+\frac{(1-p)}{(1+c \eta)^{n}}\right) e^{c^{2} \sigma^{2} / 2} \Phi(-h / \sigma+c \sigma)-K \Phi\left(-\frac{h}{\sigma}\right) \\
& \quad+p e^{-h / \eta} e^{\sigma^{2} /\left(2 \eta^{2}\right)} K \sum_{i=0}^{n-1}\left(\frac{1}{(1-c \eta)^{n-i}}-1\right)\left(\frac{\sigma}{\eta}\right)^{i} \frac{1}{\sqrt{2 \pi}} \operatorname{Hh}_{i}\left(\frac{-h \eta+\sigma^{2}}{\sigma \eta}\right) \\
& \quad+(1-p) e^{h / \eta} e^{\sigma^{2} /\left(2 \eta^{2}\right)} K \sum_{i=0}^{n-1}\left\{1-\frac{1}{(1+c \eta)^{n-i}}\right\}\left(\frac{\sigma}{\eta}\right)^{i} \frac{1}{\sqrt{2 \pi}} \operatorname{Hh}_{i}\left(\frac{h \eta+\sigma^{2}}{\sigma \eta}\right),
\end{aligned}
$$

where

$$
h=\frac{\log (K / a)-b}{c} .
$$

Proof. See the appendix.

Remark. As $\eta \rightarrow 0$, which means that the jump sizes are getting smaller and smaller, we have, thanks to (3.12),

$$
\begin{aligned}
& \frac{\sigma^{n-1}}{\eta^{n}} \frac{e^{\sigma^{2} /\left(2 \eta^{2}\right)}}{\sqrt{2 \pi}} e^{-t / \eta} \operatorname{Hh}_{n-1}\left(-\frac{t \eta-\sigma^{2}}{\sigma \eta}\right) \\
\sim & \frac{\sigma^{n-1}}{\eta^{n}} \frac{e^{\sigma^{2} /\left(2 \eta^{2}\right)}}{\sqrt{2 \pi}} e^{-t / \eta}\left(\frac{-t}{\sigma}+\frac{\sigma}{\eta}\right)^{-n} \exp \left\{-\frac{1}{2}\left(\frac{-t}{\sigma}+\frac{\sigma}{\eta}\right)^{2}\right\} \\
= & \frac{1}{\sigma \sqrt{2 \pi}}\left(\frac{-t \eta}{\sigma^{2}}+1\right)^{-n} \exp \left\{-\frac{t^{2}}{2 \sigma^{2}}\right\} \\
\rightarrow & \frac{1}{\sigma \sqrt{2 \pi}} \exp \left\{-\frac{t^{2}}{2 \sigma^{2}}\right\} ;
\end{aligned}
$$

in other words,

$$
\frac{\sigma^{n-1}}{\eta^{n}} \frac{e^{\sigma^{2} /\left(2 \eta^{2}\right)}}{\sqrt{2 \pi}} e^{-t / \eta} \mathrm{Hh}_{n-1}\left(-\frac{t \eta-\sigma^{2}}{\sigma \eta}\right) \rightarrow f_{Y}(t), \quad \text { as } \eta \rightarrow 0
$$


which is the density function of $Y$. Similarly, as $\eta \rightarrow 0$,

$$
\frac{\sigma^{n-1}}{\eta^{n}} \frac{e^{\sigma^{2} /\left(2 \eta^{2}\right)}}{\sqrt{2 \pi}} e^{t / \eta} \operatorname{Hh}_{n-1}\left(\frac{t \eta+\sigma^{2}}{\sigma \eta}\right) \rightarrow f_{Y}(t), \quad \text { as } \eta \rightarrow 0 .
$$

Therefore, taking the limit in (3.17) yields

$$
f_{X+Y}(t) \rightarrow f_{Y}(t), \quad \text { as } \eta \rightarrow 0
$$

the limit in (3.18)

$$
P(X+Y \geq u) \rightarrow \Phi\left(-\frac{u}{\sigma}\right)=P(Y \geq u), \quad \text { as } \eta \rightarrow 0,
$$

via (3.20) and (3.21); and the limit in (3.19)

$$
E\left(a e^{b+c(X+Y)}-K\right)^{+} \rightarrow a e^{b} e^{c^{2} \sigma^{2} / 2} \Phi(-h / \sigma+c \sigma)-K \Phi\left(-\frac{h}{\sigma}\right)=E\left(a e^{b+c Y}-K\right)^{+},
$$

via (3.20) and (3.21); all as we expect what should be.

\section{Option Pricing}

\subsection{European call and put options}

Theorem 1. The price of a European call option in (3.1) is given by

$$
\begin{aligned}
& \psi_{c}(0)=\sum_{n=1}^{\infty} \sum_{j=1}^{n} e^{-\lambda T} \frac{(\lambda T)^{n}}{n !} \frac{2^{j}}{2^{2 n-1}}\left(\begin{array}{c}
2 n-j-1 \\
n-1
\end{array}\right) \cdot \\
& \cdot\left\{S(0) e^{-\lambda \zeta T+n \kappa} \frac{1}{2}\left(\frac{1}{(1-\eta)^{j}}+\frac{1}{(1+\eta)^{j}}\right) \Phi\left(a_{+}\right)-e^{-r T} K \Phi\left(a_{-}\right)\right. \\
&+ \frac{1}{2} e^{-r T} e^{-h / \eta} e^{\sigma^{2} T /\left(2 \eta^{2}\right)} K \sum_{i=0}^{j-1}\left(\frac{1}{(1-\eta)^{j-i}}-1\right)\left(\frac{\sigma \sqrt{T}}{\eta}\right)^{i} \frac{1}{\sqrt{2 \pi}} \operatorname{Hh}_{i}\left(c_{-}\right) \\
&+\left.\frac{1}{2} e^{-r T} e^{h / \eta} e^{\sigma^{2} T /\left(2 \eta^{2}\right)} K \sum_{i=0}^{j-1}\left\{1-\frac{1}{(1+\eta)^{j-i}}\right\}\left(\frac{\sigma \sqrt{T}}{\eta}\right)^{i} \frac{1}{\sqrt{2 \pi}} \operatorname{Hh}_{i}\left(c_{+}\right)\right\} \\
&+ e^{-\lambda T}\left\{S(0) e^{-\lambda \zeta T} \Phi\left(b_{+}\right)-K e^{-r T} \Phi\left(b_{-}\right)\right\}
\end{aligned}
$$

where

$$
\begin{aligned}
& a_{ \pm}=\frac{\log (S(0) / K)+\left(r \pm \frac{\sigma^{2}}{2}-\lambda \zeta\right) T+n \kappa}{\sigma \sqrt{T}} \\
& b_{ \pm}=\frac{\log (S(0) / K)+\left(r \pm \frac{\sigma^{2}}{2}-\lambda \zeta\right) T}{\sigma \sqrt{T}}
\end{aligned}
$$




$$
\begin{aligned}
c_{ \pm} & =\frac{\sigma \sqrt{T}}{\eta} \pm \frac{h}{\sigma \sqrt{T}} \\
h & =\log (K / S(0))+\lambda \zeta T-\left(r-\frac{\sigma^{2}}{2}\right) T-n \kappa \\
\zeta & =\frac{e^{\kappa}}{1-\eta^{2}}-1 .
\end{aligned}
$$

Proof. See the appendix.

Remark. There are two special cases worthy of mentioning.

Case 1. Suppose $\kappa=0$. As $\eta \rightarrow 0$, which means that the jump sizes are getting smaller and smaller, the formula converges to the celebrated Black-Scholes formula. In fact, as $\eta \rightarrow 0$, we have $\zeta=\frac{1}{1-\eta^{2}}-1 \rightarrow 0$, and, by the dominated convergence theorem (the theorem is applicable because the two terms involving summations over $i$ can be, up to a constant, bounded from above by $\eta$, via (3.20) and (3.21), and we have the fact that $\left.\sum_{n=1}^{\infty} n \eta e^{-\lambda T} \frac{(\lambda T)^{n}}{n !}=\lambda T \eta \rightarrow 0\right)$,

$$
\begin{aligned}
\psi_{c}(0) \rightarrow & \sum_{n=1}^{\infty} \sum_{j=1}^{n} e^{-\lambda T} \frac{(\lambda T)^{n}}{n !} \frac{2^{j}}{2^{2 n-1}}\left(\begin{array}{c}
2 n-j-1 \\
n-1
\end{array}\right)\left\{S(0) \Phi\left(a_{+}\right)-e^{-r T} K \Phi\left(a_{-}\right)\right\} \\
& \quad+e^{-\lambda T}\left\{S(0) \Phi\left(b_{+}\right)-K e^{-r T} \Phi\left(b_{-}\right)\right\} \\
= & \sum_{n=1}^{\infty} e^{-\lambda T} \frac{(\lambda T)^{n}}{n !}\left\{S(0) \Phi\left(a_{+}\right)-e^{-r T} K \Phi\left(a_{-}\right)\right\} \frac{1}{2^{2 n-1}} \sum_{j=1}^{n} 2^{j}\left(\begin{array}{c}
2 n-j-1 \\
n-1
\end{array}\right) \\
= & \quad e^{-\lambda T}\left\{S(0) \Phi\left(b_{+}\right)-K e^{-r T} \Phi\left(b_{-}\right)\right\} \\
= & S(0) \Phi\left(b_{+}^{\prime}\right)-e^{-\lambda T} \frac{(\lambda T)^{n}}{n !}\left\{S(0) \Phi\left(b_{+}^{\prime}\right)-e^{-r T} K \Phi\left(b_{-}^{\prime}\right)\right\}+e^{-\lambda T}\left\{S(0) \Phi\left(b_{+}^{\prime}\right)-K e^{-r T} \Phi\left(b_{-}^{\prime}\right)\right\}
\end{aligned}
$$

where we have used the identity

$$
2^{2 n-1}=\sum_{k=1}^{n} 2^{k}\left(\begin{array}{c}
2 n-k-1 \\
n-1
\end{array}\right)
$$

which will be proved in the appendix at the end of the proof of Proposition 1, and the fact that in this special case

$$
a_{ \pm}=b_{ \pm}=b_{ \pm}^{\prime}:=\frac{\log (S(0) / K)+\left(r \pm \frac{\sigma^{2}}{2}\right) T}{\sigma \sqrt{T}} .
$$

Case 2. $\lambda=0$, which means that there is no jumps at all. Then

$$
\psi_{c}(0)=S(0) \Phi\left(b_{+}^{\prime}\right)-K e^{-r T} \Phi\left(b_{-}^{\prime}\right)
$$


which is the Black-Scholes formula, where

$$
b_{ \pm}^{\prime}=\frac{\log (S(0) / K)+\left(r \pm \frac{\sigma^{2}}{2}\right) T}{\sigma \sqrt{T}} .
$$

Remark. About the put-call parity.

$$
\begin{aligned}
\text { Put }- \text { Call } & =e^{-r T} \mathrm{E}^{*}\left((K-S(T))^{+}-(S(T)-K)^{+}\right) \\
& =e^{-r T} \mathrm{E}^{*}(K-S(T)) \\
& =K e^{-r T}-S(0)
\end{aligned}
$$

via (3.2), from which the price of a put option can be obtained from the formula for the call option.

Remark. About hedging. As we mentioned, the market is basically incomplete, and the usual hedging arguments are not applicable here. However, as pointed out by Merton (1976), assuming the jump risks are non-systematic and independent for different stocks, it is still possible to use the traditional delta hedging to get an asymptotic riskless hedging portfolio, as, by the law of large numbers, the unhedged risks will cancel each other asymptotically.

Remark. About programming. In mathematica and many other software packages, it is better to convert the sum into matrix operations. A mathematica code can be downloaded from the author's web page.

Remark. Although the pricing formulae involve infinite series, numerically the expressions can be evaluated very quickly to a high degree of accuracy through truncation. Our experience shows that numerically only the first 10 terms in the infinite series are needed for most applications.

\subsection{Pricing of options on futures}

Assume, for now, the term structure of interest rate is flat, and $r$ is a constant. Then the futures price, $F\left(t, T^{*}\right)$, with maturity at time $T^{*}$, is given by

$$
F\left(t, T^{*}\right)=e^{r\left(T^{*}-t\right)} S(t)
$$

Theorem 2. The price of a European call option on a futures contract is given by

$$
\begin{gathered}
\psi_{c}(D, F(0, T), K, T, \kappa, \eta, \lambda, \sigma) \\
=D \cdot\left(\sum_{n=1}^{\infty} \sum_{j=1}^{n} e^{-\lambda T} \frac{(\lambda T)^{n}}{n !} \frac{2^{j}}{2^{2 n-1}}\left(\begin{array}{c}
2 n-j-1 \\
n-1
\end{array}\right) .\right.
\end{gathered}
$$




$$
\begin{aligned}
\cdot\left\{F\left(0, T^{*}\right) e^{-\lambda \zeta T+n \kappa} \frac{1}{2}\left(\frac{1}{(1-\eta)^{j}}+\frac{1}{(1+\eta)^{j}}\right) \Phi\left(a_{+}\right)-K \Phi\left(a_{-}\right)\right. \\
+\frac{1}{2} e^{-h / \eta} e^{\sigma^{2} T /\left(2 \eta^{2}\right)} K \sum_{i=0}^{j-1}\left(\frac{1}{(1-\eta)^{j-i}}-1\right)\left(\frac{\sigma \sqrt{T}}{\eta}\right)^{i} \frac{1}{\sqrt{2 \pi}} \mathrm{Hh}_{i}\left(c_{-}\right) \\
\left.+\frac{1}{2} e^{h / \eta} e^{\sigma^{2} T /\left(2 \eta^{2}\right)} K \sum_{i=0}^{j-1}\left\{1-\frac{1}{(1+\eta)^{j-i}}\right\}\left(\frac{\sigma \sqrt{T}}{\eta}\right)^{i} \frac{1}{\sqrt{2 \pi}} \mathrm{Hh}_{i}\left(c_{+}\right)\right\} \\
\left.+e^{-\lambda T}\left\{F\left(0, T^{*}\right) e^{-\lambda \zeta T} \Phi\left(b_{+}\right)-K \Phi\left(b_{-}\right)\right\}\right),
\end{aligned}
$$

where

$$
\begin{aligned}
a_{ \pm} & =\frac{\log \left(F\left(0, T^{*}\right) / K\right) \pm \sigma^{2} T / 2-\lambda \zeta T+n \kappa}{\sigma \sqrt{T}}, \\
b_{ \pm} & =\frac{\log \left(F\left(0, T^{*}\right) / K\right) \pm \sigma^{2} T / 2-\lambda \zeta T}{\sigma \sqrt{T}} \\
c_{ \pm} & =\frac{\sigma \sqrt{T}}{\eta} \pm \frac{h}{\sigma \sqrt{T}}, \\
h & =\log \left(K / F\left(0, T^{*}\right)\right)+\lambda \zeta T+\frac{\sigma^{2} T}{2}-n \kappa, \\
\zeta & =\frac{e^{\kappa}}{1-\eta^{2}}-1 \\
D & =e^{-r T} .
\end{aligned}
$$

This theorem can be proved easily by using Theorem 1 and standard proofs about the futures options under jump diffusion models (see, for example, Musiela and Rutkowski, 1997, and Birge and Kou, 1999). Hence the proof is omitted. Notice, however, we cannot simply prove Theorem 2 by plunging in the futures formula into Theorem 1, as the underlying asset now is the futures contract rather than the spot asset itself.

Remark. Again for the following two special cases, the formula degenerates to the Black's futures option formula: case $1, \kappa=0$ and $\eta \rightarrow 0$, which means that the jump sizes are getting smaller and smaller; and case $2, \lambda=0$, which means that there is no jumps at all. Recall that the Black's formula is

$$
\psi_{c}(0)=e^{-r T}\left\{S(0) \Phi\left(b_{+}^{\prime}\right)-K \Phi\left(b_{-}^{\prime}\right)\right\}
$$

where

$$
b_{ \pm}^{\prime}=\frac{\log \left(F\left(0, T^{*}\right) / K\right) \pm \sigma^{2} T / 2}{\sigma \sqrt{T}} .
$$

Remark. We can also have a similar put-call parity for the futures options:

$$
\text { Put }- \text { Call }=e^{-r T}\left(K-F\left(0, T^{*}\right)\right) .
$$




\section{Pricing of interest rate derivatives and "volatility smile"}

In this section, we first derive closed form solution for the prices of some interest rate derivatives, particularly interest rate caps and floors, in the double exponential jump model of the previous sections; and then we will use the formulae and a real data set of interest rate caplets to show that the model is capable to produce "volatility smile".

Interest rates with jumps have been studied by many papers. Equilibrium asset pricing models for interest rates with jumps include Ahn and Thompson (1988), Attari (1996), Das and Foresi (1996), Nietert (1997); the pricing of interest rate derivatives in the presence of jumps is considered in Björk, Kabanov, and Runggaldier (1997), Burnetas and Ritchken (1997), Das and Foresi (1996), Duffie and Kan (1996), Duffie, Pan, and Singleton (1998), Jarrow and Madan (1995), and Shirakawa (1991). Various sources of jumps in interest rates, including moves by central banks, are investigated in Babbs and Webber (1997), Das (1998), El-Jahel, Lindberg, and Perraudin (1997), and Honoré (1998). In addition, the possibility of default (as modeled in Duffie and Singleton, 1996, and Jarrow and Turnbull, 1995) provides further motivation for including jumps, though we do not consider credit risk here.

In particular, a general framework of pricing interest rate caps and floors with jump risk has been studied in detail in Glasserman and Kou (1999) (see also Jamshidian, 1999). The results in this section is a corollary of that paper, with the logarithm of the jump sizes being specialized to be double exponentially distributed, except that we have to do the explicit calculation in the case of the double exponential distribution. Therefore, we only give a sketch of the results, and refer the readers to that paper for details.

\subsection{Pricing interest rate caps and floors}

Interest rate caps and floors are among the most liquated of all interest rate derivatives. To study them, it is necessary to consider the term structures based on simple forward rates with a fixed accural period $\delta$ expressed as a fraction of a year; for example, $\delta=1 / 4$ means three month rates. With $\delta$ fixed, we denote by $L(t, T)$ the forward rate for the interval from $T$ to $T+\delta$ as of time $t \leq T$. Thus, a party entering into a contract at time $t$ to borrow $\$ 1$ over the interval $[T, T+\delta]$ will receive $\$ 1$ at time $T$ and will pay to the lender $\$(1+\delta L(t, T))$ at time $T+\delta$. Mathematically speaking, the forward rate is defined as

$$
L(t, T)=\frac{1}{\delta}\left(\frac{B(t, T)}{B(t, T+\delta)}-1\right)
$$


where $B(t, \tau)$ is the time- $t$ price of a zero coupon bond maturing at time $\tau$. An interest rate caplet for the period $\left[T_{n}, T_{n+1}\right]$, with $T_{n}=n \delta$, and struck at $K$ is a derivative security paying $\delta\left(L_{n}\left(T_{n}\right)-K\right)^{+}$at time $T_{n+1}$, where $L_{n}(t)=L(t, n \delta)$.

Under the marked-point-processes model in Glasserman and Kou (1999), particularly equation (9) in that paper with $\log X_{n}^{(i)}$ being double exponential random variables instead of being normal, we can explicitly find the time- $t$ price $C_{n}(t)$ of the $n$th caplet if (25) in Glasserman and Kou (1999) holds with $f_{n}$ a double exponential density. In particular, if $\sigma_{k}$ are deterministic then the time- $t$ price of the $n$th caplet, $t<T_{n}$, is given by

$$
C_{n}(t)=\delta \psi_{c}\left(B_{n+1}(t), L_{n}(t), K, T_{n}-t, \kappa_{n}, \eta_{n}, \lambda_{n}, \sigma_{n}\right)
$$

where we have used the same notation as in Theorem 2. Obviously, for $T_{n} \leq t \leq T_{n+1}$,

$$
C_{n}(t)=\delta B_{n+1}(t)\left(L_{n}\left(T_{n}\right)-K\right)^{+}
$$

Here $\kappa_{n}, \eta_{n}, \lambda_{n}$, and $\sigma_{n}$ are risk-neutral parameters associated with the $n$th caplet. In other words, one can price interest rate caplets by using the formulae for call options on the futures contracts.

By summing the prices of individual caplets one can price a cap, which is simply a portfolio of caplets with consecutive maturities. Similarly, one can price an interest rate floor by summing the prices of single-period floors, via the formulae for put options on futures contracts.

Remark. As noted in, for example, Hull (1999), Miltersen, Sandmann, and Sondermann (1997), Glasserman and Kou (1999), from the prices of caps and floors it is possible to derive prices of puts and calls on zero coupon bonds, provided the maturity of the bond is one period later in the tenor structure than the expiration of the option. In particular, a put option on the bond struck at $K$ can be valued as a portfolio of $K$ caplets struck at $(1-K) /(\delta K)$.

\section{2. "Implied volatility smile"}

To illustrate that this model can produce "implied volatility smile", we consider a real data used first in Andersen and Andreasen (1999) for 2-year and 9-year caplets in the Japanese LIBOR market as of late May 1998. In this example, we choose a set of model parameters, calculate caplet prices at a range of strikes using (5.1), and then find the corresponding implied volatilities based on the Black's formula. More precisely, these implied volatilities are the values of $\sigma_{T}$ that equate the price computed using the Black's formula to the price computed using (5.1) with all other parameters held fixed. Their implied volatilities based on mid-market prices 


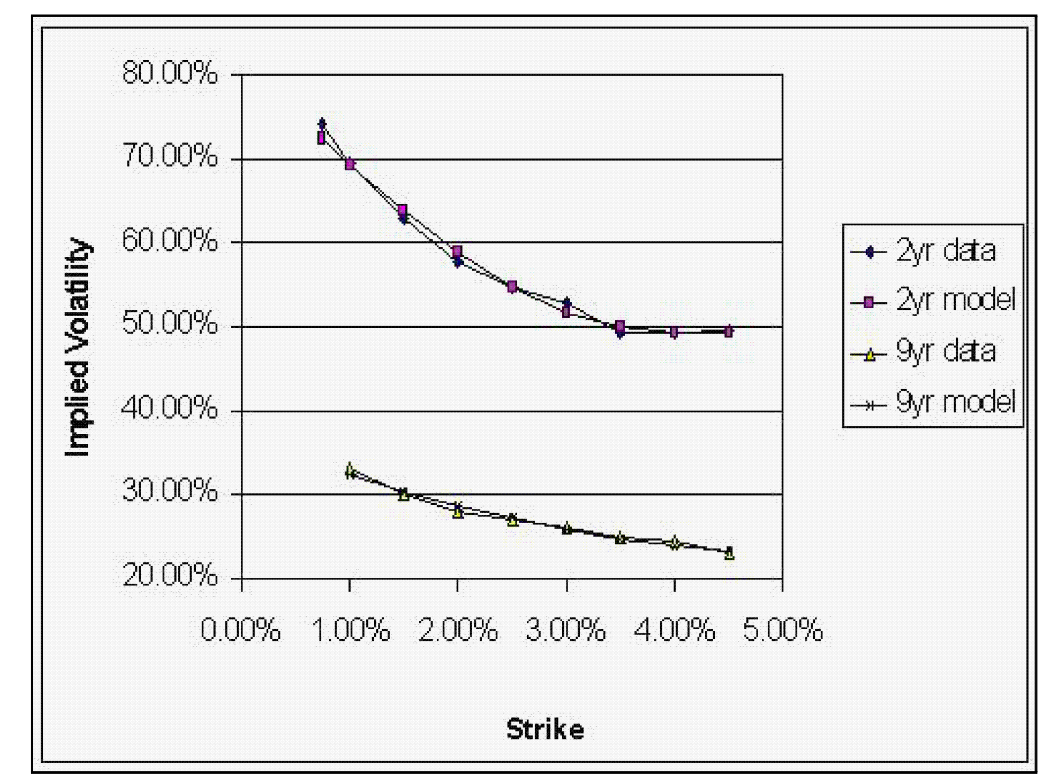

Figure 5.1: Mid-market and model implied volatilities for Japanese LIBOR caplets in May 1998. The parameters used for the model implied volatilities are $\kappa_{4}=-0.83, \eta_{4}=0.35, \lambda_{4}=0.78$, $\sigma_{4}=0.21$, and $\kappa_{18}=-0.63, \eta_{18}=0.45, \lambda_{18}=0.14, \sigma_{18}=0.08$.

are reproduced in Figure 5.1. Corresponding 6-month forward rates for the same period are $1.181 \%$ for the 2-year maturity and $2.913 \%$ for the 9-year maturity. ${ }^{1}$ Figure 5.1 also shows implied volatility curves derived from (5.1) using the same forward rates and the parameters in the caption. (With 6-month accrual intervals, the 2-year and 9-year caplets correspond to $n=4$ and $n=18$ in the notation of (5.1).) The figure suggests the possibility of a very close fit even to a very sharp market skew. Because the parameters used in (5.1) apply under a risk-neutral measure rather than the physical measure, we can interpret the parameters in Figure 5.1 as suggesting that the market attaches a large risk premium to the possibility of a sharp downward movement in Japanese interest rates. A similar interpretation is also given in Glasserman and Kou (1999) for the normal jump model.

Remark. The good fitness of the model to the market data of implied volatilities largely comes from a large number of free parameters. This is particular useful if the observed implied volatility smile is truly a "curved smile", which means that it has a right tail going upwards significantly rather than being flat as in Figure 5.1. Although the "curved smile" is frequently

\footnotetext{
${ }^{1}$ We thank Leif Andersen of General Re Financial Products for providing these values and also the mid-market implied volatilities in the figure.
} 
observed in markets, it is quite difficult for other models with fewer parameters, such as the $\mathrm{CEV}$, to fit the curve. Of course, more data would be needed to fit the models with more parameters. So our model is more appropriate for more liquated options. In addition, in actually trying to fit a model to market data, one might also want to impose additional restrictions on the parameters.

Remark. It should be pointed out that many other models can fit the volatility smile well. For the particular caplet data used here, both the CEV model (see Andersen and Andreasen, 99) and Merton normal jump model (see Glasserman and Kou, 1999) can lead to very similar

fit. A key difference is that the double exponential jump model not only can incorporate volatility smile, but also has leptokurtic feature and analytical tractability for many other options, particularly for exotic options.

\section{Advantage and disadvantage of the model}

Three major advantages of the model are that it can lead to

- leptokurtic feature, namely, compared to the geometric Brownian motion, the model can produce the desirable features of higher peak and (asymmetric) heavier tails for the underlying asset;

- "implied volatility smile", which is a puzzle for the Black-Scholes model;

- analytical tractability, namely because of the simple setting of the model, which only consists of a Brownian part and a Poisson jump part with the logarithm of the jump sizes being double exponential, it can lead to analytic solutions for various option pricing problems; in addition, the parameters in the model can be easily interpreted.

Although there are, as we discussed before, many models can incorporate at least one of the three, leptokurtic feature, "volatility smile", and analytically tractability, the current model can incorporate all of them under a unified framework.

The closed form solutions are made possible because of the simplicity of the exponential distribution and the memoryless property. In a forthcoming paper, closed form solutions for various exotic options, such as perpetual American options, barrier and lookback options will be presented. These options cannot be priced in closed form if the logarithm of the jump sizes have a normal distribution, because of the overshot problem related to the boundary crossing problems. 
One disadvantage is that the formulae, though being analytic, for option pricing appear to be long. However, this may not a major problem because the $H h$ function can be computed easily (in fact a short code would be sufficient in many software packages), and what appears to be lengthy to human eyes might make little difference in terms of computer programming, as long as it is a closed form solution.

More serious criticisms are (a). whether such a model can be incorporated in an equilibrium framework; (b) empirical justification of the model. Hopefully this paper will generate interest for further researches on both equilibrium and empirical aspects of the model. 


\section{Appendix: Proofs}

To prove Proposition 1, the following lemma is needed.

Lemma 1. Suppose $\xi_{i}$ and $\tilde{\xi}_{i}$ are independent identically distributed exponential random variables with mean $\kappa$. Then

$\sum_{i=1}^{n} \xi_{i}-\sum_{j=1}^{m} \tilde{\xi}_{j}+(n-m) \kappa \stackrel{d}{=}\left\{\begin{array}{ll}\sum_{i=1}^{k} \xi_{i}, & \text { with prob. }(1 / 2)^{n-k+m} \cdot\left(\begin{array}{c}n+m-k-1 \\ m-1\end{array}\right), k=1, \ldots, n \\ -\sum_{i=1}^{l} \xi_{i}, & \text { with prob. }(1 / 2)^{n-l+m} \cdot\left(\begin{array}{c}n+m-l-1 \\ n-1\end{array}\right), l=1, \ldots, m\end{array}\right\}$.

Proof. Introduce the random variables $A(n, m)=\sum_{i=1}^{n} \xi_{i}-\sum_{j=1}^{m} \tilde{\xi}_{j}+(n-m) \kappa$. Then

$$
A(n, m) \stackrel{d}{=}\left\{\begin{array}{ll}
A(n-1, m-1)+\xi_{n}, & 1 / 2 \\
A(n-1, m-1)-\xi_{n}, & 1 / 2
\end{array}\right\} \stackrel{d}{=}\left\{\begin{array}{cc}
A(n, m-1), & 1 / 2 \\
A(n-1, m), & 1 / 2
\end{array}\right\},
$$

via (3.4). Now image a plane with the horizontal axis representing the number of $\xi_{i}$ and vertical axis representing the number of $\tilde{\xi}_{j}$. Suppose we have a random walk on the integer lattice point of this plane. Starting from any point $(n, m), n, m \geq 1$, the random walk goes either one step to the left or one step down with equal probability $1 / 2$, and the random walk will stop once it reaches either the horizontal or the vertical axis. For any path that leading point $(n, m)$ to $(k, 0), 1 \leq k \leq n$, it must reach $(k, 1)$ first before it makes a final step to $(k, 0)$. Furthermore, all the paths going from $(n, m)$ to $(k, 1)$ must have exactly $n-k$ left's and $m-1$ down's, whence the total number of such paths is $\left(\begin{array}{c}n-k+m-1 \\ m-1\end{array}\right)$. Similarly, the total number of paths that leading point $(n, m)$ to $(0, l), 1 \leq l \leq m$, is $\left(\begin{array}{c}n-l+m-1 \\ n-1\end{array}\right)$. Thus,

$$
A(n, m) \stackrel{d}{=}\left\{\begin{array}{ll}
\sum_{i=1}^{k} \xi_{i}, & \text { with prob. }(1 / 2)^{n-k+m} \cdot\left(\begin{array}{c}
n-k+m-1 \\
m-1
\end{array}\right), k=1, \ldots, n \\
-\sum_{i=1}^{l} \xi_{i}, & \text { with prob. }(1 / 2)^{n-l+m} \cdot\left(\begin{array}{c}
n-l+m-1 \\
n-1
\end{array}\right), l=1, \ldots, m
\end{array}\right\},
$$

and the lemma is proven.

Proof of Proposition 1. It is enough to consider the case $\kappa=0$. By the same analogy used in Lemma 1 , to compute probability $p_{k}, 1 \leq k \leq n$, the probability weight assigned to $\sum_{i=1}^{k} \xi_{i}$ when we decompose $\sum_{i=1}^{n} X_{i}$, it is equivalent to consider the probability of the random walk ever reach $(k, 0)$ starting from the diagonal points $(i, n-i), 0 \leq i \leq n$, with probability of starting from $(i, n-i)$ being $\left(\begin{array}{c}n \\ i\end{array}\right) \frac{1}{2^{n}}$. Notice that the point $(k, 0)$ can only be reached from points $(i, n-i)$, with the constraint $k \leq i \leq n-1$, because the random walk stops once it reaches the horizontal axis. Therefore, for $1 \leq k \leq n-1$, (A.1) leads to

$$
p_{k}=\sum_{i=k}^{n-1} P(\text { going from }(i, n-i) \text { to }(k, 0)) \cdot P(\text { starting from }(i, n-i))
$$




$$
\begin{aligned}
& =\sum_{i=k}^{n-1}\left(\frac{1}{2}\right)^{i+(n-i)-k}\left(\begin{array}{c}
i+(n-i)-k-1 \\
(n-i)-1
\end{array}\right)\left(\begin{array}{c}
n \\
i
\end{array}\right) \frac{1}{2^{n}} \\
& =\sum_{i=k}^{n-1}\left(\frac{1}{2}\right)^{n-k}\left(\begin{array}{c}
n-k-1 \\
n-i-1
\end{array}\right)\left(\begin{array}{c}
n \\
i
\end{array}\right) \frac{1}{2^{n}} \\
& =\sum_{i=k}^{n-1}\left(\frac{1}{2}\right)^{n-k}\left(\begin{array}{c}
n-k-1 \\
i-k
\end{array}\right)\left(\begin{array}{c}
n \\
i
\end{array}\right) \frac{1}{2^{n}},
\end{aligned}
$$

where $\left(\begin{array}{l}0 \\ 0\end{array}\right)$ is defined to be one. Letting $j=i-k$ yields, for $1 \leq k \leq n-1$,

$$
\begin{aligned}
p_{k} & =\sum_{j=0}^{n-k-1}\left(\frac{1}{2}\right)^{n-k}\left(\begin{array}{c}
n-k-1 \\
j
\end{array}\right)\left(\begin{array}{c}
n \\
j+k
\end{array}\right) \frac{1}{2^{n}} \\
& =\frac{2^{k}}{2^{2 n}} \sum_{j=0}^{n-k-1}\left(\begin{array}{c}
n-k-1 \\
j
\end{array}\right)\left(\begin{array}{c}
n \\
n-j-k
\end{array}\right) .
\end{aligned}
$$

But we have the combinatorial identity

$$
\sum_{j=0}^{n-k-1}\left(\begin{array}{c}
n-k-1 \\
j
\end{array}\right)\left(\begin{array}{c}
n \\
n-j-k
\end{array}\right)=\left(\begin{array}{c}
2 n-k-1 \\
n-k
\end{array}\right) .
$$

Thus, for $1 \leq k \leq n-1$,

$$
p_{k}=\frac{2^{k}}{2^{2 n}}\left(\begin{array}{c}
2 n-k-1 \\
n-k
\end{array}\right)
$$

Of course,

$$
p_{n}=\frac{1}{2^{n}}
$$

In summary, we have

$$
p_{k}=\frac{2^{k}}{2^{2 n}}\left(\begin{array}{c}
2 n-k-1 \\
n-k
\end{array}\right)=\frac{2^{k}}{2^{2 n}}\left(\begin{array}{c}
2 n-k-1 \\
n-1
\end{array}\right), \quad 1 \leq k \leq n
$$

Similarly, to compute $p_{-k}, k \geq 1$, which is the probability weight assigned to $-\sum_{i=1}^{k} \xi_{i}$ when we decompose $\sum_{i=1}^{n} X_{i}$, it is equivalent to consider the probability of the random walk ever reach $(0, k)$ from the diagonal points $(i, n-i), 0 \leq i \leq n$. This is given by

$$
\begin{aligned}
p_{-k} & =\sum_{i=k}^{n-1} P(\text { going from }(n-i, i) \text { to }(0, k)) \cdot P(\text { starting from }(n-i, i)) \\
& =\sum_{i=k}^{n-1}\left(\frac{1}{2}\right)^{(n-i)+i-k}\left(\begin{array}{c}
(n-i)+i-k-1 \\
(n-i)-1
\end{array}\right)\left(\begin{array}{c}
n \\
n-i
\end{array}\right) \frac{1}{2^{n}} \\
& =\sum_{i=k}^{n-1}\left(\frac{1}{2}\right)^{n-k}\left(\begin{array}{c}
n-k-1 \\
n-i-1
\end{array}\right)\left(\begin{array}{c}
n \\
i
\end{array}\right) \frac{1}{2^{n}} \\
& =p_{k} .
\end{aligned}
$$


Therefore, we can introduce a random variable $M(n)$, such that

$$
P(M(n)=k)=2 p_{k}=\frac{2^{k}}{2^{2 n-1}}\left(\begin{array}{c}
2 n-k-1 \\
n-1
\end{array}\right), \quad 1 \leq k \leq n,
$$

and the decomposition is, for $n \geq 2$,

$$
\sum_{i=1}^{n} X_{i} \stackrel{d}{=}\left\{\begin{array}{cc}
\sum_{i=1}^{M(n)} \xi_{i}, & \text { with probability } 1 / 2 \\
-\sum_{i=1}^{M(n)} \xi_{i}, & \text { with probability } 1 / 2
\end{array}\right\},
$$

where $\xi_{i}$ are i.i.d. exponential random variables. Incidentally, we have also shown that

$$
2^{2 n-1}=\sum_{k=1}^{n} 2^{k}\left(\begin{array}{c}
2 n-k-1 \\
n-1
\end{array}\right)
$$

because $\sum\left(p_{k}+p_{-k}\right)=1$, and thus $M(n)$ is a proper discrete distribution with the domain $\{1,2, \ldots, n\}$.

Proof of Proposition 2. Case 1. $x \rightarrow \infty$. We will prove it by induction. Clearly it is true for $n=-1$. Now suppose it is true for $n=k$. Then

$$
\operatorname{Hh}_{k}(x) \sim \frac{1}{x^{k+1}} e^{-x^{2} / 2},
$$

which means that for every $\epsilon>0$ it must be that for all large enough $x$

$$
1-\epsilon \leq \operatorname{Hh}_{k}(x) /\left\{\frac{1}{x^{k+1}} e^{-x^{2} / 2}\right\} \leq 1+\epsilon .
$$

Therefore, for all large $x$, we have the following bounds on $\mathrm{Hh}_{k+1}(x)$ :

$$
(1-\epsilon) \int_{x}^{\infty} \frac{1}{y^{k+1}} e^{-y^{2} / 2} d y \leq \operatorname{Hh}_{k+1}(x)=\int_{x}^{\infty} \operatorname{Hh}_{k}(y) d y \leq(1+\epsilon) \int_{x}^{\infty} \frac{1}{y^{k+1}} e^{-y^{2} / 2} d y
$$

But, as $x \rightarrow \infty$,

$$
\int_{x}^{\infty} \frac{1}{y^{k+1}} e^{-y^{2} / 2} d y=2^{-1-\frac{1}{2} k} \cdot \Gamma\left(-\frac{1}{2} k, \frac{1}{2} x^{2}\right) \sim \frac{1}{x^{k+2}} e^{-x^{2} / 2} .
$$

Therefore

$$
\lim _{x \rightarrow \infty} \operatorname{Hh}_{k+1}(x) /\left\{\frac{1}{x^{k+2}} e^{-x^{2} / 2}\right\}=1
$$

and the result is proven.

Case 2. $x \rightarrow-\infty$. It is clearly true for $n=-1$. So we only study the situation when $n \geq 0$. As $x \rightarrow-\infty$

$$
\begin{aligned}
\operatorname{Hh}_{n}(x) & =\frac{1}{n !} \int_{x}^{\infty}(t-x)^{n} e^{-t^{2} / 2} d t \\
& \leq \frac{2^{n}}{n !} \int_{x}^{\infty}\left(|t|^{n}+|x|^{n}\right) e^{-t^{2} / 2} d t \\
& \leq \frac{2^{n}}{n !} \int_{-\infty}^{\infty}|t|^{n} e^{-t^{2} / 2} d t+\frac{2^{n}}{n !} \int_{-\infty}^{\infty}|x|^{n} e^{-t^{2} / 2} d t \\
& =O\left(|x|^{n}\right),
\end{aligned}
$$


and the proof is terminated.

Proof of Proposition 3. We shall study it in serval cases.

Case 1 , when $\beta<0$ and $\alpha>0$. Then there is nothing more to say as the integral is $\infty$, because the integrand goes to infinity as $x \rightarrow \infty$.

Case 2 , when $\beta>0$. In this case, we must have, for $n \geq 0$,

$$
e^{\alpha x} \operatorname{Hh}_{n}(\beta x-\delta) \rightarrow 0, \text { as } x \rightarrow \infty,
$$

for any constant $\alpha$, thanks to (3.12). Integration by parts leads to

$$
\begin{aligned}
I_{n} & =\int_{\lambda}^{\infty} e^{\alpha x} \operatorname{Hh}_{n}(\beta x-\delta) d x \\
& =\frac{1}{\alpha} \int_{\lambda}^{\infty} \operatorname{Hh}_{n}(\beta x-\delta) d e^{\alpha x} \\
& =\frac{1}{\alpha}\left[\left.\operatorname{Hh}_{n}(\beta x-\delta) e^{\alpha x}\right|_{x=\lambda} ^{\infty}-\int_{\lambda}^{\infty} e^{\alpha x} d \operatorname{Hh}_{n}(\beta x-\delta)\right] \\
& =-\frac{1}{\alpha} \operatorname{Hh}_{n}(\beta \lambda-\delta) e^{\alpha \lambda}+\frac{\beta}{\alpha} \int_{\lambda}^{\infty} e^{\alpha x} \operatorname{Hh}_{n-1}(\beta x-\delta) d x
\end{aligned}
$$

where the limiting behavior in (A.2) has been applied. In other words, we have a recursion, for $n \geq 0$,

$$
I_{n}=-\frac{e^{\alpha \lambda}}{\alpha} \operatorname{Hh}_{n}(\beta \lambda-\delta)+\frac{\beta}{\alpha} I_{n-1}
$$

with the initial condition

$$
\begin{aligned}
I_{-1} & =\int_{\lambda}^{\infty} e^{\alpha x} \mathrm{Hh}_{-1}(\beta x-\delta) d x \\
& =\sqrt{2 \pi} \int_{\lambda}^{\infty} e^{\alpha x} \varphi(-\beta x+\delta) d x \\
& =\frac{\sqrt{2 \pi}}{\beta} \exp \left\{\frac{\alpha \delta}{\beta}+\frac{\alpha^{2}}{2 \beta^{2}}\right\} \Phi\left(-\beta \lambda+\delta+\frac{\alpha}{\beta}\right) .
\end{aligned}
$$

Solving it yields, for $n \geq-1$,

$$
\begin{aligned}
I_{n} & =-\frac{e^{\alpha \lambda}}{\alpha} \sum_{i=0}^{n}\left(\frac{\beta}{\alpha}\right)^{i} \operatorname{Hh}_{n-i}(\beta \lambda-\delta)+\left(\frac{\beta}{\alpha}\right)^{n+1} I_{-1} \\
& =-\frac{e^{\alpha \lambda}}{\alpha} \sum_{i=0}^{n}\left(\frac{\beta}{\alpha}\right)^{n-i} \operatorname{Hh}_{i}(\beta \lambda-\delta)+\left(\frac{\beta}{\alpha}\right)^{n+1} \frac{\sqrt{2 \pi}}{\beta} \exp \left\{\frac{\alpha \delta}{\beta}+\frac{\alpha^{2}}{2 \beta^{2}}\right\} \Phi\left(-\beta \lambda+\delta+\frac{\alpha}{\beta}\right),
\end{aligned}
$$

where the sum over an empty set is defined to be zero.

Case 3, when $\beta<0$ and $\alpha<0$. In this case, we must also have, for $n \geq 0$,

$$
e^{\alpha x} \operatorname{Hh}_{n}(\beta x-\delta) \rightarrow 0, \text { as } x \rightarrow \infty
$$


for any constant $\alpha<0$, thanks to (3.13). Using integration by parts and (A.3), we again have a recursion, for $n \geq 0$,

$$
I_{n}=-\frac{e^{\alpha \lambda}}{\alpha} \operatorname{Hh}_{n}(\beta \lambda-\delta)+\frac{\beta}{\alpha} I_{n-1}
$$

but with a different initial condition

$$
\begin{aligned}
I_{-1} & =\int_{\lambda}^{\infty} e^{\alpha x} \mathrm{Hh}_{-1}(\beta x-\delta) d x \\
& =\sqrt{2 \pi} \int_{\lambda}^{\infty} e^{\alpha x} \varphi(-\beta x+\delta) d x \\
& =-\frac{\sqrt{2 \pi}}{\beta} \exp \left\{\frac{\alpha \delta}{\beta}+\frac{\alpha^{2}}{2 \beta^{2}}\right\} \Phi\left(\beta \lambda-\delta-\frac{\alpha}{\beta}\right) .
\end{aligned}
$$

Solving it yields, for $n \geq-1$,

$$
\begin{aligned}
I_{n} & =-\frac{e^{\alpha \lambda}}{\alpha} \sum_{i=0}^{n}\left(\frac{\beta}{\alpha}\right)^{i} \operatorname{Hh}_{n-i}(\beta \lambda-\delta)+\left(\frac{\beta}{\alpha}\right)^{n+1} I_{-1} \\
& =-\frac{e^{\alpha \lambda}}{\alpha} \sum_{i=0}^{n}\left(\frac{\beta}{\alpha}\right)^{n-i} \operatorname{Hh}_{i}(\beta \lambda-\delta)-\left(\frac{\beta}{\alpha}\right)^{n+1} \frac{\sqrt{2 \pi}}{\beta} \exp \left\{\frac{\alpha \delta}{\beta}+\frac{\alpha^{2}}{2 \beta^{2}}\right\} \Phi\left(\beta \lambda-\delta-\frac{\alpha}{\beta}\right),
\end{aligned}
$$

where the sum over an empty set is again defined to be zero. The proof is terminated.

Proof of Proposition 4. We prove it in three cases.

Case 1 . The density of $X+Y$. We have

$$
\begin{gathered}
f_{X+Y}(t) \\
=\int_{-\infty}^{\infty} f_{X}(t-x) f_{Y}(x) d x \\
=p \int_{-\infty}^{t} \frac{(1 / \eta) e^{-(t-x) / \eta}((t-x) / \eta)^{n-1}}{(n-1) !} \frac{1}{\sigma \sqrt{2 \pi}} e^{-x^{2} /\left(2 \sigma^{2}\right)} d x \\
\quad+(1-p) \int_{t}^{\infty} \frac{(1 / \eta) e^{(t-x) / \eta}((x-t) / \eta)^{n-1}}{(n-1) !} \frac{1}{\sigma \sqrt{2 \pi}} e^{-x^{2} /\left(2 \sigma^{2}\right)} d x \\
=p e^{-t / \eta}\left(1 / \eta^{n}\right) \int_{-\infty}^{t} \frac{e^{x / \eta}(t-x)^{n-1}}{(n-1) !} \frac{1}{\sigma \sqrt{2 \pi}} e^{-x^{2} /\left(2 \sigma^{2}\right)} d x \\
\quad+(1-p) e^{t / \eta}\left(1 / \eta^{n}\right) \int_{t}^{\infty} \frac{e^{-x / \eta}(x-t)^{n-1}}{(n-1) !} \frac{1}{\sigma \sqrt{2 \pi}} e^{-x^{2} /\left(2 \sigma^{2}\right)} d x \\
=p e^{-t / \eta}\left(1 / \eta^{n}\right) e^{\sigma^{2} /\left(2 \eta^{2}\right)} \int_{-\infty}^{t} \frac{(t-x)^{n-1}}{(n-1) !} \frac{1}{\sigma \sqrt{2 \pi}} e^{-\left(x-\sigma^{2} / \eta\right)^{2} /\left(2 \sigma^{2}\right)} d x \\
\quad+(1-p) e^{t / \eta}\left(1 / \eta^{n}\right) e^{\sigma^{2} /\left(2 \eta^{2}\right)} \int_{t}^{\infty} \frac{(x-t)^{n-1}}{(n-1) !} \frac{1}{\sigma \sqrt{2 \pi}} e^{-\left(x+\sigma^{2} / \eta\right)^{2} /\left(2 \sigma^{2}\right)} d x .
\end{gathered}
$$

By letting $y=\left(x-\sigma^{2} / \eta\right) / \sigma$, and $\tilde{y}=\left(x+\sigma^{2} / \eta\right) / \sigma$, we have

$$
f_{X+Y}(t)
$$




$$
\begin{aligned}
= & p e^{-t / \eta} e^{\sigma^{2} /\left(2 \eta^{2}\right)}\left(1 / \eta^{n}\right) \int_{-\infty}^{t / \sigma-\sigma / \eta} \frac{\left(t-\sigma y-\sigma^{2} / \eta\right)^{n-1}}{(n-1) !} \frac{1}{\sqrt{2 \pi}} e^{-y^{2} / 2} d y \\
& +(1-p) e^{t / \eta} e^{\sigma^{2} /\left(2 \eta^{2}\right)}\left(1 / \eta^{n}\right) \int_{t / \sigma+\sigma / \eta}^{\infty} \frac{\left(\sigma \tilde{y}-\sigma^{2} / \eta-t\right)^{n-1}}{(n-1) !} \frac{1}{\sqrt{2 \pi}} e^{-\tilde{y}^{2} / 2} d y \\
= & p e^{-t / \eta} e^{\sigma^{2} /\left(2 \eta^{2}\right)} \sigma^{n-1} / \eta^{n} \int_{-\infty}^{t / \sigma-\sigma / \eta} \frac{(t / \sigma-y-\sigma / \eta)^{n-1}}{(n-1) !} \frac{1}{\sqrt{2 \pi}} e^{-y^{2} / 2} d y \\
& \quad+(1-p) e^{t / \eta} e^{\sigma^{2} /\left(2 \eta^{2}\right)} \sigma^{n-1} / \eta^{n} \int_{t / \sigma+\sigma / \eta}^{\infty} \frac{(\tilde{y}-\sigma / \eta-t / \sigma)^{n-1}}{(n-1) !} \frac{1}{\sqrt{2 \pi}} e^{-\tilde{y}^{2} / 2} d y \\
= & \frac{e^{\sigma^{2} /\left(2 \eta^{2}\right)}}{\sqrt{2 \pi}}\left(\sigma^{n-1} / \eta^{n}\right)\left\{p e^{-t / \eta} \mathrm{Hh}_{n-1}(-t / \sigma+\sigma / \eta)+(1-p) e^{t / \eta} \operatorname{Hh}_{n-1}(t / \sigma+\sigma / \eta)\right\},
\end{aligned}
$$

because

$$
\begin{aligned}
\frac{1}{(n-1) !} \int_{-\infty}^{a}(a-y)^{n-1} \frac{e^{-y^{2} / 2}}{\sqrt{2 \pi}} d y & =\frac{1}{(n-1) !} \int_{-a}^{\infty}\left(a+y_{1}\right)^{n-1} \frac{e^{-y_{1}^{2} / 2}}{\sqrt{2 \pi}} d y_{1} \\
& =\frac{1}{\sqrt{2 \pi}} \operatorname{Hh}_{n-1}(-a),
\end{aligned}
$$

and similarly

$$
\frac{1}{(n-1) !} \int_{b}^{\infty}(y-b)^{n-1} \frac{1}{\sqrt{2 \pi}} e^{-y^{2} / 2} d y=\frac{1}{\sqrt{2 \pi}} \operatorname{Hh}_{n-1}(b),
$$

where $y_{1}=-y$, and the proof of Case 1 is terminated.

Case 2. $P(X+Y \geq u)$. We have, via the density given in (3.17), that

$$
\begin{aligned}
& P(X+Y \geq u)=\int_{u}^{\infty} f_{X+Y}(t) d t \\
= & \frac{\sigma^{n}}{\eta^{n}} \frac{e^{\sigma^{2} /\left(2 \eta^{2}\right)}}{\sigma \sqrt{2 \pi}}\left\{p \int_{u}^{\infty} e^{-t / \eta} \operatorname{Hh}_{n-1}\left(-\frac{t \eta-\sigma^{2}}{\sigma \eta}\right) d t+(1-p) \int_{u}^{\infty} e^{t / \eta} \operatorname{Hh}_{n-1}\left(\frac{t \eta+\sigma^{2}}{\sigma \eta}\right) d t\right\} .
\end{aligned}
$$

Substituting $\beta=(-1 / \sigma)<0, \alpha=(-1 / \eta)<0, \delta=(-\sigma / \eta)$, and $\lambda=u$ in (3.15) yields

$$
\begin{aligned}
& \int_{u}^{\infty} e^{-t / \eta} \operatorname{Hh}_{n-1}\left(-\frac{t \eta-\sigma^{2}}{\sigma \eta}\right) d t \\
&=\eta e^{-u / \eta} \sum_{i=0}^{n-1}(\eta / \sigma)^{n-1-i} \operatorname{Hh}_{i}(-u / \sigma+\sigma / \eta) \\
& \quad+(\eta / \sigma)^{n} \sigma \sqrt{2 \pi} \exp \left\{-\sigma^{2} / \eta^{2}+\frac{\sigma^{2}}{2 \eta^{2}}\right\} \Phi(-u / \sigma+\sigma / \eta-\sigma / \eta) \\
&=\eta e^{-u / \eta} \sum_{i=0}^{n-1}(\eta / \sigma)^{n-1-i} \operatorname{Hh}_{i}(-u / \sigma+\sigma / \eta)+(\eta / \sigma)^{n} \sigma \sqrt{2 \pi} \exp \left\{-\frac{\sigma^{2}}{2 \eta^{2}}\right\} \Phi(-u / \sigma) ;
\end{aligned}
$$

and substituting $\beta=(1 / \sigma)>0, \alpha=(1 / \eta), \delta=(-\sigma / \eta)$, and $\lambda=u$ in (3.14) yields

$$
\int_{u}^{\infty} e^{t / \eta} \mathrm{Hh}_{n-1}\left(\frac{t \eta+\sigma^{2}}{\sigma \eta}\right) d t
$$




$$
\begin{aligned}
=-\eta e^{u / \eta} \sum_{i=0}^{n-1}\left(\frac{\eta}{\sigma}\right)^{n-1-i} \operatorname{Hh}_{i}(u / \sigma+\sigma / \eta) \\
\quad+\left(\frac{\eta}{\sigma}\right)^{n} \sigma \sqrt{2 \pi} \exp \left\{-\sigma^{2} / \eta^{2}+\frac{\sigma^{2}}{2 \eta^{2}}\right\} \Phi(-u / \sigma-\sigma / \eta+\sigma / \eta) \\
=(-\eta) e^{u / \eta} \sum_{i=0}^{n-1}\left(\frac{\eta}{\sigma}\right)^{n-1-i} \operatorname{Hh}_{i}(u / \sigma+\sigma / \eta)+\left(\frac{\eta}{\sigma}\right)^{n} \sigma \sqrt{2 \pi} \exp \left\{-\frac{\sigma^{2}}{2 \eta^{2}}\right\} \Phi(-u / \sigma) .
\end{aligned}
$$

Therefore,

$$
\begin{aligned}
& P(X+Y \geq u) \\
= & \frac{\sigma^{n}}{\eta^{n}} \frac{1}{\sigma \sqrt{2 \pi}} e^{\sigma^{2} /\left(2 \eta^{2}\right)}\left\{p\left[\eta e^{-u / \eta} \sum_{i=0}^{n-1}\left(\frac{\eta}{\sigma}\right)^{n-1-i} \operatorname{Hh}_{i}\left(\frac{-u \eta+\sigma^{2}}{\sigma \eta}\right)+\left(\frac{\eta}{\sigma}\right)^{n} \sigma \sqrt{2 \pi} e^{-\sigma^{2} /\left(2 \eta^{2}\right)} \Phi(-u / \sigma)\right]\right. \\
& \left.+(1-p)\left[-\eta e^{u / \eta} \sum_{i=0}^{n-1}\left(\frac{\eta}{\sigma}\right)^{n-1-i} \operatorname{Hh}_{i}\left(\frac{u \eta+\sigma^{2}}{\sigma \eta}\right)+\left(\frac{\eta}{\sigma}\right)^{n} \sigma \sqrt{2 \pi} e^{-\sigma^{2} /\left(2 \eta^{2}\right)} \Phi(-u / \sigma)\right]\right\} \\
= & e^{\sigma^{2} /\left(2 \eta^{2}\right)}\left\{p\left[e^{-u / \eta} \sum_{i=0}^{n-1}\left(\frac{\eta}{\sigma}\right)^{-i} \frac{1}{\sqrt{2 \pi}} \operatorname{Hh}_{i}\left(\frac{-u \eta+\sigma^{2}}{\sigma \eta}\right)+e^{-\sigma^{2} /\left(2 \eta^{2}\right)} \Phi\left(-\frac{u}{\sigma}\right)\right]\right. \\
& \left.\quad+(1-p)\left[-e^{u / \eta} \sum_{i=0}^{n-1}\left(\frac{\eta}{\sigma}\right)^{-i} \frac{1}{\sqrt{2 \pi}} \operatorname{Hh}_{i}\left(\frac{u \eta+\sigma^{2}}{\sigma \eta}\right)+e^{-\sigma^{2} /\left(2 \eta^{2}\right)} \Phi\left(-\frac{u}{\sigma}\right)\right]\right\},
\end{aligned}
$$

and the proof of Case 2 is terminated.

Case 3. $E\left(a e^{b+c(X+Y)}-K\right)^{+}$. By using the density given in (3.17), we get, for $n \geq 1$

$$
\begin{aligned}
& E\left(a e^{b+c(X+Y)}-K\right)^{+} \\
= & \int_{h}^{\infty}\left(a e^{b+c t}-K\right) f_{X+Y}(t) d t \\
=a e^{b} \int_{h}^{\infty} e^{c t} f_{X+Y}(t) d t-K P(X+Y \geq h) & a e^{b} \frac{\sigma^{n}}{\eta^{n}} \frac{1}{\sigma \sqrt{2 \pi}} e^{\sigma^{2} /\left(2 \eta^{2}\right)}\left\{p \int_{h}^{\infty} e^{c t} e^{-t / \eta} \operatorname{Hh}_{n-1}\left(-\frac{t \eta-\sigma^{2}}{\sigma \eta}\right) d u\right. \\
+ & \left.(1-p) \int_{h}^{\infty} e^{c t} e^{t / \eta} \mathrm{Hh}_{n-1}\left(\frac{t \eta+\sigma^{2}}{\sigma \eta}\right) d u\right\} \\
& \quad-K \Phi\left(-\frac{h}{\sigma}\right)-K p e^{-h / \eta} e^{\sigma^{2} /\left(2 \eta^{2}\right)} \sum_{i=0}^{n-1}\left(\frac{\sigma}{\eta}\right)^{i} \frac{1}{\sqrt{2 \pi}} \operatorname{Hh}_{i}\left(\frac{-h \eta+\sigma^{2}}{\sigma \eta}\right) \\
& +K(1-p) e^{h / \eta} e^{\sigma^{2} /\left(2 \eta^{2}\right)} \sum_{i=0}^{n-1}\left(\frac{\sigma}{\eta}\right)^{i} \frac{1}{\sqrt{2 \pi}} \operatorname{Hh}_{i}\left(\frac{h \eta+\sigma^{2}}{\sigma \eta}\right),
\end{aligned}
$$

via (3.18), where

$$
h=\frac{\log (K / a)-b}{c} .
$$


Substituting $\beta=(-1 / \sigma)<0, \alpha=(c-1 / \eta)<0, \delta=(-\sigma / \eta)$, and $\lambda=h$ in $(3.15)$

$$
\begin{aligned}
& \int_{h}^{\infty} e^{c t} e^{-t / \eta} \operatorname{Hh}_{n-1}\left(-\frac{t \eta-\sigma^{2}}{\sigma \eta}\right) d t \\
&=-\frac{e^{(c-1 / \eta) h}}{c-1 / \eta} \sum_{i=0}^{n-1}\left(-\frac{1}{(c-1 / \eta) \sigma}\right)^{n-1-i} \operatorname{Hh}_{i}(-h / \sigma+\sigma / \eta) \\
&-\left(\frac{-1}{(c-1 / \eta) \sigma}\right)^{n} \sqrt{2 \pi}(-\sigma) \exp \left\{(c-1 / \eta) \sigma^{2} / \eta+\frac{(c-1 / \eta)^{2} \sigma^{2}}{2}\right\} \Phi(-h / \sigma+\sigma / \eta+(c-1 / \eta) \sigma) \\
&= \frac{\eta e^{(c-1 / \eta) h}}{1-c \eta} \sum_{i=0}^{n-1}\left(\frac{\eta}{(1-c \eta) \sigma}\right)^{n-1-i} \operatorname{Hh}_{i}\left(\frac{-h \eta+\sigma^{2}}{\sigma \eta}\right) \\
&+\sigma\left(\frac{\eta}{(1-c \eta) \sigma}\right)^{n} \sqrt{2 \pi} \exp \left\{\frac{1}{2} c^{2} \sigma^{2}-\frac{1}{2} \sigma^{2} / \eta^{2}\right\} \Phi(-h / \sigma+c \sigma),
\end{aligned}
$$

and substituting $\beta=1 / \sigma>0, \alpha=(c+1 / \eta), \delta=(-\sigma / \eta)$, and $\lambda=h$ in (3.14)

$$
\begin{aligned}
& \int_{h}^{\infty} e^{c t} e^{t / \eta} \operatorname{Hh}_{n-1}\left(\frac{t \eta+\sigma^{2}}{\sigma \eta}\right) d t \\
=- & \frac{e^{(c+1 / \eta) h}}{c+1 / \eta} \sum_{i=0}^{n-1}\left(\frac{1}{(c+1 / \eta) \sigma}\right)^{n-1-i} \operatorname{Hh}_{i}(h / \sigma+\sigma / \eta) \\
& +\left(\frac{1}{(c+1 / \eta) \sigma}\right)^{n} \sigma \sqrt{2 \pi} \exp \left\{(c+1 / \eta)(-\sigma / \eta) \sigma+\frac{(c+1 / \eta)^{2} \sigma^{2}}{2}\right\} \Phi(-h / \sigma-\sigma / \eta+(c+1 / \eta) \sigma) \\
=- & \frac{\eta e^{(c+1 / \eta) h}}{1+c \eta} \sum_{i=0}^{n-1}\left(\frac{\eta}{(c \eta+1) \sigma}\right)^{n-1-i} \operatorname{Hh}_{i}\left(\frac{h \eta+\sigma^{2}}{\sigma \eta}\right) \\
& +\left(\frac{\eta}{(c \eta+1) \sigma}\right)^{n} \sigma \sqrt{2 \pi} \exp \left\{\frac{1}{2} c^{2} \sigma^{2}-\frac{1}{2} \sigma^{2} / \eta^{2}\right\} \Phi(-h / \sigma+c \sigma) .
\end{aligned}
$$

Therefore,

$$
\begin{aligned}
E\left(a e^{b+c(X+Y)}-K\right)^{+} & =a e^{b} \frac{1}{\sqrt{2 \pi}} e^{\sigma^{2} /\left(2 \eta^{2}\right)}\left\{p \frac{e^{(c-1 / \eta) h}}{1-c \eta} \sum_{i=0}^{n-1}\left(\frac{1}{(1-c \eta)}\right)^{n-1-i}\left(\frac{\eta}{\sigma}\right)^{-i} \operatorname{Hh}_{i}\left(\frac{-h \eta+\sigma^{2}}{\sigma \eta}\right)\right. \\
+ & \frac{p \sqrt{2 \pi}}{(1-c \eta)^{n}} \exp \left\{\frac{1}{2} c^{2} \sigma^{2}-\frac{1}{2} \sigma^{2} / \eta^{2}\right\} \Phi(-h / \sigma+c \sigma) \\
& -(1-p) \frac{\eta e^{(c+1 / \eta) h}}{1+c \eta} \sum_{i=0}^{n-1}\left(\frac{1}{(c \eta+1)}\right)^{n-1-i}\left(\frac{\eta}{\sigma}\right)^{-i} \operatorname{Hh}_{i}\left(\frac{h \eta+\sigma^{2}}{\sigma \eta}\right) \\
+ & \left.\frac{(1-p) \sqrt{2 \pi}}{(c \eta+1)^{n}} \exp \left\{\frac{1}{2} c^{2} \sigma^{2}-\frac{1}{2} \sigma^{2} / \eta^{2}\right\} \Phi(-h / \sigma+c \sigma)\right\} \\
& -K \Phi\left(-\frac{h}{\sigma}\right)-K p e^{-h / \eta} e^{\sigma^{2} /\left(2 \eta^{2}\right)} \sum_{i=0}^{n-1}\left(\frac{\sigma}{\eta}\right)^{i} \frac{1}{\sqrt{2 \pi}} \operatorname{Hh}_{i}\left(\frac{-h \eta+\sigma^{2}}{\sigma \eta}\right)
\end{aligned}
$$




$$
+K(1-p) e^{h / \eta} e^{\sigma^{2} /\left(2 \eta^{2}\right)} \sum_{i=0}^{n-1}\left(\frac{\sigma}{\eta}\right)^{i} \frac{1}{\sqrt{2 \pi}} \mathrm{Hh}_{i}\left(\frac{h \eta+\sigma^{2}}{\sigma \eta}\right) .
$$

After some algebra, we get

$$
\begin{gathered}
E\left(a e^{b+c(X+Y)}-K\right)^{+} \\
=p e^{-h / \eta} e^{\sigma^{2} /\left(2 \eta^{2}\right)} \sum_{i=0}^{n-1}\left(\frac{a e^{b+c h}}{(1-c \eta)^{n-i}}-K\right)\left(\frac{\sigma}{\eta}\right)^{i} \frac{1}{\sqrt{2 \pi}} \operatorname{Hh}_{i}\left(\frac{-h \eta+\sigma^{2}}{\sigma \eta}\right) \\
-K \Phi\left(-\frac{h}{\sigma}\right)+a e^{b}\left(\frac{p}{(1-c \eta)^{n}}+\frac{(1-p)}{(1+c \eta)^{n}}\right) e^{c^{2} \sigma^{2} / 2} \Phi(-h / \sigma+c \sigma) \\
+(1-p) e^{h / \eta} e^{\sigma^{2} /\left(2 \eta^{2}\right)} \sum_{i=0}^{n-1}\left\{-\frac{a e^{b+c h}}{(c \eta+1)^{n-i}}+K\right\}\left(\frac{\sigma}{\eta}\right)^{i} \frac{1}{\sqrt{2 \pi}} \operatorname{Hh}_{i}\left(\frac{h \eta+\sigma^{2}}{\sigma \eta}\right) .
\end{gathered}
$$

Since $e^{b+c h}=(K / a)$, we get

$$
\begin{gathered}
E\left(a e^{b+c(X+Y)}-K\right)^{+} \\
=p e^{-h / \eta} e^{\sigma^{2} /\left(2 \eta^{2}\right)} K \sum_{i=0}^{n-1}\left(\frac{1}{(1-c \eta)^{n-i}}-1\right)\left(\frac{\sigma}{\eta}\right)^{i} \frac{1}{\sqrt{2 \pi}} \operatorname{Hh}_{i}\left(\frac{-h \eta+\sigma^{2}}{\sigma \eta}\right) \\
+a e^{b}\left(\frac{p}{(1-c \eta)^{n}}+\frac{(1-p)}{(1+c \eta)^{n}}\right) e^{c^{2} \sigma^{2} / 2} \Phi(-h / \sigma+c \sigma)-K \Phi\left(-\frac{h}{\sigma}\right) \\
+(1-p) e^{h / \eta} e^{\sigma^{2} /\left(2 \eta^{2}\right)} K \sum_{i=0}^{n-1}\left\{1-\frac{1}{(1+c \eta)^{n-i}}\right\}\left(\frac{\sigma}{\eta}\right)^{i} \frac{1}{\sqrt{2 \pi}} \operatorname{Hh}_{i}\left(\frac{h \eta+\sigma^{2}}{\sigma \eta}\right) .
\end{gathered}
$$

and the proof is terminated.

Proof of Theorem 1. Since $N(t)$ has a Poisson distribution with mean $\lambda t$, conditioning on $N(t)$, we have

$$
\begin{aligned}
\psi_{c}(0)= & \sum_{n=0}^{\infty} \mathrm{E}^{*}\left(e^{-r T}\left(S(0) \exp \left\{\left(r-\frac{\sigma^{2}}{2}-\lambda \zeta\right) T+\sigma \sqrt{T} Z\right\} \prod_{j=1}^{N(T)} V_{j}-K\right)^{+} \mid N(T)=n\right) . \\
& \cdot P(N(T)=n) \\
= & e^{-r T} \sum_{n=0}^{\infty} e^{-\lambda T} \frac{(\lambda T)^{n}}{n !} \mathrm{E}^{*}\left(S(0) e^{-\lambda \zeta T} \exp \left\{\left(r-\frac{\sigma^{2}}{2}\right) T+\sigma \sqrt{T} Z\right\} \prod_{j=1}^{n} V_{j}-K\right)^{+} \\
= & e^{-r T} \sum_{n=0}^{\infty} e^{-\lambda T} \frac{(\lambda T)^{n}}{n !} \mathrm{E}^{*}\left(S(0) e^{-\lambda \zeta T} \exp \left\{\left(r-\frac{\sigma^{2}}{2}\right) T+\sigma \sqrt{T} Z+\sum_{j=1}^{n} X_{j}\right\}-K\right)^{+}
\end{aligned}
$$

where recalling that $V_{j}=e^{X_{i}}$ and $\prod$ over the empty set is set to be one.

By the decomposition (3.7), we have, for $n \geq 1$, the following decomposition

$$
\sum_{i=1}^{n} X_{i} \stackrel{d}{=} n \kappa+\sum_{i=1}^{M(n)} Y_{i}
$$


where $Y_{i}$ are i.i.d. double random variables

$$
Y_{i}=\left\{\begin{array}{ll}
\xi_{i}, & \text { with probability } 1 / 2 \\
-\xi_{i}, & \text { with probability } 1 / 2
\end{array}\right\}
$$

$\xi_{i}$ being exponential random variable with mean $\eta$, and $M(n)$ is a discrete random variable with

$$
P(M(n)=j)=\frac{2^{j}}{2^{2 n-1}}\left(\begin{array}{c}
2 n-j-1 \\
n-1
\end{array}\right), \quad 1 \leq j \leq n .
$$

Notice that $M(n)$ is independent of all other random variables.

Therefore, conditioning on $M(n)$,

$$
\begin{gathered}
\psi_{c}(0) \\
=e^{-r T} \sum_{n=0}^{\infty} e^{-\lambda T} \frac{(\lambda T)^{n}}{n !} \mathrm{E}^{*}\left(S(0) e^{-\lambda \zeta T} \exp \left\{\left(r-\frac{\sigma^{2}}{2}\right) T+\sigma \sqrt{T} Z+n \kappa+\sum_{i=1}^{M(n)} Y_{i}\right\}-K\right)^{+} \\
=e^{-r T} \sum_{n=1}^{\infty} \sum_{j=1}^{n} e^{-\lambda T} \frac{(\lambda T)^{n}}{n !} \frac{2^{j}}{2^{2 n-1}}\left(\begin{array}{c}
2 n-j-1 \\
n-1
\end{array}\right) \cdot \\
\cdot \mathrm{E}^{*}\left(S(0) e^{-\lambda \zeta T} \exp \left\{\left(r-\frac{\sigma^{2}}{2}\right) T+\sigma \sqrt{T} Z+n \kappa+\sum_{i=1}^{j} Y_{i}\right\}-K\right)^{+} \\
+e^{-r T} e^{-\lambda T} \mathrm{E}^{*}\left(e^{-r T}\left(S(0) e^{-\lambda \zeta T} \exp \left\{\left(r-\frac{\sigma^{2}}{2}\right) T+\sigma \sqrt{T} Z\right\}-K\right)^{+}\right) .
\end{gathered}
$$

Now substituting $a=S(0) e^{-\lambda \zeta T}, b=\left(r-\frac{\sigma^{2}}{2}\right) T+n \kappa, p=1 / 2$, and $c=1$ into (3.19), we have for $0<\eta<1$,

$$
\begin{gathered}
\mathrm{E}^{*}\left(S(0) e^{-\lambda \zeta T} \exp \left\{\left(r-\frac{\sigma^{2}}{2}\right) T+\sigma \sqrt{T} Z+n \kappa+\sum_{i=1}^{j} Y_{i}\right\}-K\right)^{+} \\
=S(0) e^{-\lambda \zeta T+\left(r-\frac{\sigma^{2}}{2}\right) T+n \kappa} \frac{1}{2}\left(\frac{1}{(1-\eta)^{j}}+\frac{1}{(1+\eta)^{j}}\right) e^{\sigma^{2} T / 2} \Phi(-h /(\sigma \sqrt{T})+\sigma \sqrt{T})-K \Phi\left(-\frac{h}{\sigma \sqrt{T}}\right) \\
+\frac{1}{2} e^{-h / \eta} e^{\sigma^{2} T /\left(2 \eta^{2}\right)} K \sum_{i=0}^{j-1}\left(\frac{1}{(1-\eta)^{j-i}}-1\right)\left(\frac{\sigma \sqrt{T}}{\eta}\right)^{i} \frac{1}{\sqrt{2 \pi}} \operatorname{Hh}_{i}\left(\frac{-h \eta+\sigma^{2} T}{(\sigma \sqrt{T}) \eta}\right) \\
+\frac{1}{2} e^{h / \eta} e^{\sigma^{2} T /\left(2 \eta^{2}\right)} K \sum_{i=0}^{j-1}\left\{1-\frac{1}{(1+\eta)^{j-i}}\right\}\left(\frac{\sigma \sqrt{T}}{\eta}\right)^{i} \frac{1}{\sqrt{2 \pi}} \operatorname{Hh}_{i}\left(\frac{h \eta+\sigma^{2} T}{(\sigma \sqrt{T}) \eta}\right),
\end{gathered}
$$

with

$$
h=\frac{\log (K / a)-b}{c}=\log (K / S(0))+\lambda \zeta T-\left(r-\frac{\sigma^{2}}{2}\right) T-n \kappa .
$$


Furthermore, it is easy to check that

$$
\mathrm{E}^{*}\left(S(0) \exp \left\{\left(r-\frac{\sigma^{2}}{2}-\lambda \zeta\right) T+\sigma \sqrt{T} Z\right\}-K\right)^{+}=S(0) e^{(r-\lambda \zeta) T} \Phi\left(b_{+}\right)-K \Phi\left(b_{-}\right),
$$

where

$$
b_{ \pm}=\frac{\log (S(0) / K)+\left(r \pm \frac{\sigma^{2}}{2}-\lambda \zeta\right) T}{\sigma \sqrt{T}} .
$$

Thus, we have proved the theorem.

\section{References}

[1] Abramowitz, M. and Stegun, I.A. (1972). Handbook of Mathematical Function. U. S. National Bureau of Standards, 10th Printing.

[2] Ahn, C.M., and Thompson, H.E. (1988) Jump-Diffusion Processes and the Term Structure of Interest Rates, Journal of Finance, 43, 155-174.

[3] Andersen, T. (1996). Return volatility and trading volume. An information flow interpretation of stochastic volatility. Journal of Finance. Vol. 51, 169-204.

[4] Andersen, L. and Andreasen, J. (1999). Jump-diffusion processes: volatility smile fitting and numerical methods for pricing. Working paper. General Re Financial Products, New York.

[5] Attari, M. (1996). Discontinuous Interest Rate Processes: An Equilibrium Model for Bond Option Prices, working paper, University of Iowa, Iowa City.

[6] Babbs, S., and Webber, N. (1997). Term Structure Modelling under Alternative Official Regimes, in Mathematics of Derivative Securities, M.A.H. Dempster and S.R. Pliska, eds., Cambridge University Press.

[7] Barndorff-Nielsen (1998). Probability and statistics: self-decomposability, finance and turbulence. In Probability Towards 2000, edited by L. Accardi and C.C. Heyde. Springer, NY.

[8] Björk, T., Kabanov, Y., and Runggaldier, W. (1997). Bond Market Structure in the Presence of Marked Point Processes, Mathematical Finance, 7, 211-239.

[9] Black, F. (1976). The pricing of commodity contract. J. of Financial Econ., Vol. 3, 167-179.

[10] Black, F. and Scholes, M. (1973). The pricing of options and corporate liabilities. J. Polit. Econ., 81, 637-659.

[11] Blattberg, R.C. and Gonedes, N. (1974). A comparison of the stable and student distributions as statistical models for stock prices. Journal of Business. Vol 47, 244-280.

[12] Birge, J.R. and Kou, S.G. (1999). Pricing of electricity options under a jump diffusion model. Working paper, Columbia University.

[13] Boyle, P., Broadie, M., and Glasserman, P. (1997). Simulation methods for security pricing. Journal of Economic Dynamics and Control. Vol 21, 1267-1321. 
[14] Brace, A., Gatarek, D., and Musiela, M. (1997). The market model of interest rate dynamics. Math. Finance, Vol. 7, 127-155.

[15] Burnetas, A., and Ritchken, P. (1997). On Rational Jump Diffusion Models: An Approach Using Potentials, Review of Derivatives Research, 1, 325-349.

[16] Clark, P. K. (1973). A subordinated stochastic process model with finite variance for speculative prices. Econometrica. Vol. 41, 135-155.

[17] Cox, J.T., Ingersoll, J.E., and Ross, S.A. (1985). A theory of the term structure of interest rates. Econometrica. Vol 53, 385-407.

[18] Cox, J.T. and Rubinstein, M. (1985). Option Markets. Prentice Hall, Englewood Cliffs, N.J.

[19] Cox, J.T. and Ross, S.A. (1976). The valuation of options for alternative stochastic processes. Journal of Financial Economics. Vol 3, 145-166.

[20] Das, S.R. (1998). Poisson-Gaussian Processes and the Bond Markets, NBER Working Paper No. 6631.

[21] Das, S.R., and Foresi, S. (1996). Exact Solutions for Bond and Option Prices with Systematic Jump Risk, Review of Derivatives Research, 1, 7-24.

[22] Davydov, D., and Linetsky (1999). The valuation and hedging aof barrier and lookback options for alternative stochastic processes. Working paper, University of Michigan.

[23] Derman, E. and Kani, I. Riding on a smile. (1994). RISK. Feb. 32-39.

[24] Duffie, D. (1995). Dynamic Asset Pricing Theory. 2nd edn. Princeton University Press, Princeton.

[25] Duffie, D., and Kan, R. (1996). A Yield-Factor Model of Interest Rates, Mathematical Finance, 6, 379-406.

[26] Duffie, D., Pan, J., and Singleton, K. (1998). Transform Analysis and Option Pricing for Affine Jump-Diffusions, working paper, Graduate School of Business, Stanford University.

[27] Duffie, D., and Singleton, K. (1996). Modeling Term Structures of Defaultable Bonds, Working paper, Graduate School of Business, Stanford University.

[28] Dupire, B. (1994). Pricing with a smile. RISK. Feb. 18-20.

[29] Eberlein, E. and Keller, U. (1995). Hyperbolic Distributions in Finance. Bernoulli. Vol 1, 281-299.

[30] El-Jahel, L., Lindberg, H., and Perraudin, W. (1997). Interest Rate Distributions, Yield Curve Modelling and Monetary Policy, in Mathematics of Derivative Securities, M.A.H. Dempster and S.R. Pliska, eds., Cambridge University Press.

[31] Elliot, R.J. and Kopp, P.E. (1998). Mathematics of Financial Markets. Spinger, New York.

[32] Engle, R. (1982). Autoregressive conditional heteroscedasticity with estimates of the variance of U.K. inflation. Econometrica. Vol. 50. 987-1008. 
[33] Engle, R. (1995). ARCH, Selected Readings. Oxford, Oxford University Press.

[34] Fama, E. (1963). Mandelbrot and the stable Peritian hypothesis. Journal of Business, Vol $36,297-306$.

[35] Fama, E. (1965). The behavior of stock-market prices. Journal of Business, Vol 38, 34-105.

[36] Geman, H., Madan, D., and Yor, M. (1998). Asset prices in Brownian motion: only in business times. Working paper, University of Paris IX, Dauphine.

[37] Glasserman, P. and Kou, S.G. (1999). The term structure of simple interest rates with jump risk. Working paper, Columbia University.

[38] Gouriéroux, C. (1997). ARCH Models and Financial Applications. Spinger, New York.

[39] Heath, D., Jarrow, R. and Morton, A. (1992). Bond pricing and the term structure models: a new methodology for contingent claims valuation. Econometrica. Vol 60, 77-105.

[40] Heyde, C.C. (1999). A risky asset model with strong dependence through fractal activity time. Dept. of Statistics, Columbia University.

[41] Honoré, P. (1998). Modelling Interest Rate Dynamics in a Corridor with Jump Processes, working paper, Department of Finance, Aarhus School of Business, Denmark.

[42] Hull, J.C. (1999). Options, Futures, and Other Derivative Securities, 4th edition, Prentice Hall, New Jersey.

[43] Hull, J.C. and White, A. (1987). The pricing of options on assets with stochastic volatilities. Journal of Finance. Vol 42, 281-300.

[44] Hurst, S.R. and Platen, E., and Rachev, S.R. (1997). Subordinated Markov models: a comparison. Financial Engineering and Japanese Markets. Vol. 4, 97-124.

[45] Ingersoll, J.E. (1988). Theory of Financial Decision Making. Rowman \& Littlefield, Maryland.

[46] Jamshidian, F. (1997). LIBOR and swap market models and measures. Finance and Stochastic. Vol 1. 293-330.

[47] Jamshidian, F. (1999). LIBOR market model with semimartingales. Working paper, NetAnalytic Limited.

[48] Jarrow, R., and Turnbull, S. (1995). Pricing Options on Financial Securities Subject to Default Risk, Journal of Finance, 50, 53-86.

[49] Jarrow, R., and Madan, D. (1995). Option Pricing using the Term Structure of Interest Rates to Hedge Systematic Discontinuities in Asset Returns, Mathematical Finance, 5, 311-336.

[50] Johnson, N., Kotz, S., and Balakrishnan, N. (1995). Continuous Univariate Distribution, Vol. 2, 2nd ed., Wiley, New York.

[51] Karatzas, I. and Shreve, S. (1998). Methods of Mathematical Finance. Springer, New York. 
[52] Laplace, P.S. (1774). Mémoire sur la probabilité des causes par les èvénemens, Mémoires de Mathématique et de Physique, Vol. 6, 621-656.

[53] Madan, D.B. and Seneta, E. (1990). The variance gamma (V.G.) model for share market returns. Journal of Business, Vol 63, 511-524.

[54] Mandelbrot, B. (1963). The variation of certain speculative prices, Journal of Business, Vol 36, 394-419.

[55] Mandelbrot, B. (1967). The variation of some other speculative prices, Journal of Business, Vol 40, 511-524.

[56] Mandelbrot, B., Fisher, A. and Calvet, L. (1997). A multifractal model of asset returns. Cowles Foundation Discussion Paper No. 1164.

[57] Merton, R. C. (1976). Option pricing when underlying stock returns are discontinuous. J. of Financial Economics. Vol. 3, 125-144.

[58] Merton, R.C. (1990). Continuous-Time Finance. Blackwell, Cambridge.

[59] Miltersen, K., Sandmann, K., and Sondermann, D. (1997). Closed form solutions for term structure derivatives with lognormal interest rates. J. of Finance. Vol. 70, 409-430.

[60] Musiela and Rutkowski (1997). Martingale Methods in Financial Modeling. Springer, New York.

[61] Nietert, B. (1997). Jump/Diffusion Option Pricing — a Reexamination from an Economic Viewpoint, Department of Finance, Passau University, Germany.

[62] Peters, E.E. (1991). Chaos and Order in the Capital Markets. Wiley, New York.

[63] Peters, E.E. (1994). Fractal Market Analysis. Wiley, New York.

[64] Praetz, P.D. (1972). The distribution of share price changes. Journal of Business. Vol 45, 49-55.

[65] Rogers, L.C.G. (1997). Arbitrage from fractional Brownian motion. Mathematical Finance. Vol. 7, 95-105.

[66] Rubinstein, M. (1994). Implied binomial trees. Journal of Finance. Vol 49, 771-818.

[67] Samorodnitsky, G. and Taqqu, M.S. (1994). Stable Non-Gaussian Random Processes: Stochastic Models with Infinite Variance. Chapman and Hall, New York.

[68] Shanthikumar, J.G. (1985). Bilateral Phase-Type Distributions. Naval Research Logistics Quarterly. Vol. 32, 119-136.

[69] Shirakawa, H. (1991) Interest Rate Option Pricing with Poisson-Gaussian Forward Rate Curve Processes, Math. Finance, 1, 77-94.

[70] White, H. (1980). A heteroscedasticity consistent covariance matrix estimation and a direct test for heteroscedasticy. Econometrica. Vol. 48, 817-838.

[71] Willinger, W. Taqqu, M. and Teverovsky, V. (1999). Stock market prices and long-range dependence. Finance and Stochastics. Vol 3, 1-13. 